Article

\title{
Distinct Roles for RAB10 and RAB29 in Pathogenic LRRK2-Mediated Endolysosomal Trafficking Alterations
}

\author{
Pilar Rivero-Ríos ${ }^{1,2,+}$, Maria Romo-Lozano ${ }^{1,+}{ }^{+}$Belén Fernández ${ }^{1} \mathbb{D}$, Elena Fdez ${ }^{1}$ \\ and Sabine Hilfiker ${ }^{3, *}$ \\ 1 Institute of Parasitology and Biomedicine “López-Neyra”, Consejo Superior de Investigaciones \\ Científicas (CSIC), Avda del Conocimiento s/n, 18016 Granada, Spain; mriveror@umich.edu (P.R.-R.); \\ maria.romo@ipb.csic.es (M.R.-L.); belenfernandez@ipb.csic.es (B.F.); fdez@ipb.csic.es (E.F.) \\ 2 Life Sciences Institute, University of Michigan, Ann Arbor, MI 48109, USA \\ 3 Department of Anesthesiology, New Jersey Medical School, Rutgers, the State University of New Jersey, \\ Newark, NJ 07103, USA \\ * Correspondence: sabine.hilfiker@rutgers.edu \\ + These authors contributed equally.
}

Received: 23 June 2020; Accepted: 13 July 2020; Published: 17 July 2020

\begin{abstract}
Mutations in the gene encoding leucine-rich repeat kinase 2 (LRRK2) cause familial Parkinson's disease, and sequence variations are associated with the sporadic form of the disease. LRRK2 phosphorylates a subset of RAB proteins implicated in secretory and recycling trafficking pathways, including RAB8A and RAB10. Another RAB protein, RAB29, has been reported to recruit LRRK2 to the Golgi, where it stimulates its kinase activity. Our previous studies revealed that G2019S LRRK2 expression or knockdown of RAB8A deregulate epidermal growth factor receptor (EGFR) trafficking, with a concomitant accumulation of the receptor in a RAB4-positive recycling compartment. Here, we show that the G2019S LRRK2-mediated EGFR deficits are mimicked by knockdown of RAB10 and rescued by expression of active RAB10. By contrast, RAB29 knockdown is without effect, but expression of RAB29 also rescues the pathogenic LRRK2-mediated trafficking deficits independently of Golgi integrity. Our data suggest that G2019S LRRK2 deregulates endolysosomal trafficking by impairing the function of RAB8A and RAB10, while RAB29 positively modulates non-Golgi-related trafficking events impaired by pathogenic LRRK2.
\end{abstract}

Keywords: Parkinson's disease; LRRK2; RAB10; RAB29; endolysosome; Golgi

\section{Introduction}

Mutations in the LRRK2 gene are a common cause of familial Parkinson's disease (PD) and are also observed in sporadic PD patients, indicating a key role for LRRK2 across the entire disease spectrum [1-3]. LRRK2 is a large multidomain protein kinase, and the most prominent pathogenic mutation (G2019S) situated within the kinase domain increases its activity in vitro as well as in vivo, suggesting that LRRK2 inhibitors may be therapeutically beneficial for at least LRRK2-related PD [4-11].

Recent phosphoproteomic analyses identified a small subset of RAB GTPases (RAB3, RAB8, RAB10, RAB12, RAB35, and RAB43) as endogenous substrates for the LRRK2 activity [12]. RAB proteins are master regulators of all eukaryotic membrane trafficking events [13-15]. They are prenylated at their C-termini, and are delivered as well as extracted from membranes by GDI (RAB GDP dissociation inhibitor). At the membrane, their nucleotide-bound state is regulated by guanine nucleotide exchange factors (GEFs), which activate the RABs, and by GTPase activating proteins (GAPs), which return RABs into their inactive, GDP-bound state [16]. In their membrane- and GTP-bound forms, RABs recruit 
effector proteins, which bring about various events including vesicle formation, motility, and vesicle docking at the respective target membranes [13-15]. Differences in the nucleotide-bound state of RAB proteins are reflected by differences in the conformation of the highly conserved switch II region [17]. LRRK2 phosphorylates the RAB proteins on their switch II regions, which consequently interferes with their binding to most regulatory and effector proteins $[12,18,19]$. Therefore, the LRRK2-mediated phosphorylation may cause deficits in the specific membrane trafficking pathways modulated by each of these RAB proteins.

Multiple lines of evidence indicate that LRRK2 plays a role in the endolysosomal system [20], which may be related to its ability to bind and/or phosphorylate RAB proteins [12,18,21-27]. However, the precise underlying mechanisms remain poorly defined. In previous studies, we have analyzed the effect of pathogenic LRRK2 on the endocytic trafficking of the epidermal growth factor receptor (EGFR). In contrast to other receptors, the EGFR can either follow a purely endolysosomal degradative route or be recycled back to the cell surface, and these distinct fates can be triggered by distinct ligand concentrations [28,29]. Pathogenic LRRK2 expression was found to impair the endolysosomal degradative trafficking as well as the endocytic recycling of the EGFR, with a concomitant decrease in the levels of active, GTP-bound RAB7A, and an accumulation of the receptor in a RAB4-positive recycling compartment [30,31]. All alterations due to pathogenic LRRK2 expression were mimicked by knockdown of RAB8A and rescued by expression of active RAB8A, suggesting that they are mediated, at least in part, by RAB8A inactivation [31]. These findings support the notion that the phosphorylation of RAB8A by pathogenic LRRK2 may correlate with a loss-of-function phenotype.

RAB10 serves as a prominent LRRK2 kinase substrate in all cell lines and tissues analyzed [12,18,32-35]. It is widely expressed in peripheral tissues as well as in the brain, where it is present in all cell types [36], and localized to a tubular perinuclear endocytic recycling compartment partially overlapping with RAB8A [37-40]. RAB10 has been described to modulate a variety of membrane trafficking events including exocytic polarized targeting and endocytic recycling events [41], and can perform functions partially redundant with those of RAB8A [42-45]. However, the link between RAB10 and the endolysosomal trafficking deficits owing to pathogenic LRRK2 remains unknown.

RAB29 is contained within the PARK16 locus, which is linked to Parkinson's disease, even though it remains unclear how variants in this locus influence disease risk [2,46-52]. Variants within the RAB29 and the LRRK2 locus seem to impact PD in a non-additive manner [22,49,53], suggesting that the two proteins function in a common cellular pathway. In support of this notion, pathogenic G2019S LRRK2 expression causes deficits in Golgi-lysosome-related sorting events, which are mimicked by knockdown of RAB29 and rescued by RAB29 overexpression, respectively, and transcriptome and protein analyses indicate that decreased RAB29 levels correlate with increased PD risk [22]. Other studies have reported a physical interaction between LRRK2 and RAB29, and when overexpressed, RAB29 can recruit LRRK2 to the Golgi network [23,54-56]. Such recruitment stimulates the LRRK2 kinase activity and causes an accumulation of phospho-RABs in the same membrane compartment, likely because the phospho-proteins are unable to be extracted by GDI [56,57]. Thus, a controversial picture has emerged whereby RAB29 either rescues the pathogenic LRRK2-mediated phenotypes [22] or serves as a membrane-bound activator for the LRRK2 kinase activity, anticipated to exacerbate pathogenic LRRK2-mediated membrane trafficking phenotypes [1,56,57].

In this study, we investigated the link between RAB10 and RAB29 and the endolysosomal trafficking deficits mediated by G2019S LRRK2. We show that expression of active RAB10 rescues, while knockdown of RAB10 mimics the trafficking deficits mediated by G2019S LRRK2. Furthermore, RAB8A and RAB10 seem to perform functionally redundant roles, at least with respect to the trafficking of the specific receptor analyzed here. In contrast, while not required for the endolysosomal trafficking of the EGFR per se, a modest increase in RAB29 levels rescues the pathogenic LRRK2-mediated deficits. Such rescue occurs independent of Golgi integrity and independent of the Golgi localization of RAB29, highlighting the possibility that this RAB protein may play additional physiological roles important for proper endolysosomal trafficking unrelated to its proposed role as a LRRK2 activator. 


\section{Materials and Methods}

\subsection{DNA Constructs and Site-Directed Mutagenesis}

Triple flag-tagged LRRK2 constructs, as well as N-terminally tagged RAB8A, RAB10, RAB29, RAB7A, and RAB4 constructs, have been previously described [31,39,55,58]. The siRNA-resistant form of RAB10 was generated by introducing three silent mutations into the target sequence of the seed region of the RAB10-siRNA (Ambion, Thermo Fisher, Waltham, MA, USA, ID s21391), altering the original sequence by site-directed mutagenesis (QuickChange, Stratagene, San Diego, CA, USA) with primers 5'-GTCTATTCCTATGGTGGAAATAAATGTCGTGTTGAAGGCATCATCCGAAAAACGA-3' and 5'-TCGTTTTTCGGATGATGCCTTCAACACGACATTTATTTCCACCATAGGAATAGAC-3' . The identity of the construct was verified by sequencing of the entire coding region. DNA was prepared from bacterial cultures grown at $37{ }^{\circ} \mathrm{C}$ using PureYield ${ }^{\mathrm{TM}}$ Plasmid Midiprep System (Promega, Madison, WI, USA) according to the manufacturer's instructions.

\subsection{Cell Culture and Transfection}

HeLa cells (ATCC; American Tissue Culture Collection) were cultured in $100 \mathrm{~mm}$ dishes in full medium (DMEM containing 10\% fetal bovine serum, non-essential amino acids, high glucose) at $37^{\circ} \mathrm{C}$ in $5 \% \mathrm{CO}_{2}$. Confluent cells were harvested with $0.05 \%$ trypsin/0.02 mM EDTA in PBS, and subcultured at a ratio of 1:2-1:3. Cells were plated onto six-well plates and transfected the following day (70-80\% confluence) using Lipofectamine 2000 (LF2000) (Invitrogen, Carlsbad, CA, USA) for 4 h in DMEM, followed by replacement with full medium. Double-transfections were performed using $2 \mu \mathrm{g}$ of LRRK2 plasmids and $500 \mathrm{ng}$ of RAB plasmids of interest. Alternatively, and where indicated, cells were transfected using Jetprime in full medium overnight according to manufacturer's instructions using the same DNA concentrations as for transfections with LF2000. The following day, transfected cells were replated at a 1:2 ratio onto coverslips in 24-well plates, and proteins were expressed for $48 \mathrm{~h}$ before analysis.

\subsection{Knockdown of $R A B 10, R A B 8 A$, or $R A B 29$ by $R N A i$}

HeLa cells were plated into six-well plates, and transfected the following day at $70-80 \%$ confluence using $25 \mathrm{nM}$ siRNA and $4 \mu \mathrm{L}$ of jetPRIME transfection reagent (Polyplus-Transfection SA, catalog number 114-15) in $200 \mu \mathrm{L}$ of jetPRIME buffer overnight in full medium. For knockdown experiments in the presence of GFP-tagged RAB construct expression, cells were transfected with 500 ng of the indicated RAB constructs using LF2000 for $4 \mathrm{~h}$ in DMEM, followed by replacement with full medium, and transfection with siRNA using jetPRIME overnight, as indicated above. In all cases, cells were passaged the next day and processed $48 \mathrm{~h}$ after transfection. RNAi reagents included the following: Silencer Select Negative Control Number 1 siRNA (Ambion, Thermo Fisher, catalog number 4390843), Silencer Select RAB8A (Ambion, Thermo Fisher, ID s8679), Silencer Select RAB10 (Ambion, Thermo Fisher, ID s21391), and Silencer Select RAB29 (Ambion, Thermo Fisher, ID s17082). In all cases, knockdown efficacy of the various siRNA reagents was confirmed by Western blotting with the appropriate antibodies.

\subsection{Immunofluorescence and Laser Confocal Imaging}

Where indicated, HeLa cells were incubated with $5 \mu \mathrm{g} / \mathrm{mL}$ brefeldin A (BFA) (Sigma Aldrich, St. Louis, MO, USA) before fixation with $4 \%$ paraformaldehyde (PFA) in PBS for 20 min at room temperature. Cells were next washed in $0.5 \%$ Triton X-100 in PBS for $3 \times 5 \mathrm{~min}$, followed by incubation in blocking buffer (10\% goat serum, $0.5 \%$ Triton X-100 in PBS) for $1 \mathrm{~h}$. Coverslips were incubated with primary antibody in blocking buffer for $1 \mathrm{~h}$ at room temperature, followed by washes in $0.5 \%$ Triton X-100 in PBS and incubation with secondary antibodies for $1 \mathrm{~h}$. Coverslips were washed with PBS, and mounted in mounting medium with DAPI (Vector Laboratories, Burlingame, CA, USA). Primary antibodies included rabbit polyclonal anti- $\beta$-COP protein (1:200; Thermo Fisher, PA1-061) and mouse 
monoclonal anti-flag (1:500; Sigma, F1804), and secondary antibodies included Alexa594-conjugated goat anti-rabbit (1:2000; Invitrogen) or Alexa594-conjugated goat anti-mouse (1:2000; Invitrogen), respectively. To determine the localization of GFP-tagged RAB protein variants in the absence of antibody staining, cells were fixed as described above, but only briefly permeabilized with $0.5 \%$ Triton X-100 in PBS for $3 \mathrm{~min}$, followed by washes in PBS and mounting, as described above.

Images were acquired on a Leica TCS-SP5 confocal microscope using a $63 \times 1.4$ numerical aperture oil UV objective (HCX PLAPO CS). Single excitation for each wavelength separately was used for all acquisitions (488 nm argon laser line and a 500-545 nm emission band pass; $543 \mathrm{HeNe}$ laser line and a $556-673 \mathrm{~nm}$ emission band pass; $405 \mathrm{~nm}$ UV diode and a $422-466 \mathrm{~nm}$ emission band pass). The same laser settings and exposure times were employed for acquisition of individual experiments, and 10 to 15 image sections of selected areas acquired with a step size of $0.4 \mu \mathrm{m}$. All z-stack images were analyzed and processed using Fiji.

Live-cell fluorescence microscopy to determine colocalization of fluorescent EGF with GFP-RAB4 in the absence or presence of pathogenic LRRK2 or siRNA of RABs was performed as previously described. Briefly, cells were seeded onto $35 \mathrm{~mm}$ glass-bottom dishes (ibidi) $24 \mathrm{~h}$ after transfection, and were serum-starved overnight. The following day, medium was replaced by phenol-free, serum-free DMEM (Gibco), and cells incubated with $100 \mathrm{ng} / \mathrm{mL}$ Alexa647-EGF (Invitrogen) for $20 \mathrm{~min}$ at $4{ }^{\circ} \mathrm{C}$ to allow for surface binding. Excess fluorescent EGF was removed by washing cells twice in ice-cold PBS, and cells were incubated for $20 \mathrm{~min}$ at $37^{\circ} \mathrm{C}$ to allow for internalization of bound EGF. Individual z-stage images corresponding to the cell center were acquired, and the JACoP plugin of Fiji was employed for quantification of colocalization of GFP-RAB4 with Alexa647-EGF. For this purpose, images were thresholded, and the percentage of colocalization was quantified by calculating the Mander's coefficients (M1 for red channel, and percentage of colocalization calculated as M1 × 100) [31], with 15-20 independent cells analyzed per condition per experiment.

\subsection{Fluorescent EGF Binding and Uptake Assays}

Assays were performed essentially as described [31]. Briefly, transfected HeLa cells were plated onto coverslips the day after transfection, and serum-starved overnight. The following day, medium was replaced with fresh serum-free medium containing $100 \mathrm{ng} / \mathrm{mL}$ Alexa555-EGF (Invitrogen) at $4{ }^{\circ} \mathrm{C}$ to allow for ligand binding in the absence of internalization. In parallel experiments, control cells were washed with PBS, followed by acid stripping $\left(0.5 \mathrm{M} \mathrm{NaCl}, 0.2 \mathrm{M}\right.$ acetic acid, $\left.\mathrm{pH} 2.5,3 \mathrm{~min}, 4^{\circ} \mathrm{C}\right)$ to confirm that fluorescent EGF was merely surface-bound under those conditions. After binding of fluorescent EGF at $4{ }^{\circ} \mathrm{C}$, cells were washed twice in ice-cold PBS, and then transferred to prewarmed serum-free medium to allow for uptake, and cells were fixed at the indicated times (10 min and $30 \mathrm{~min}$ ) to quantify internalized fluorescent EGF. Fixation was performed with $4 \%$ PFA in PBS for $15 \mathrm{~min}$ at room temperature, cells were softly permeabilized with $0.5 \%$ Triton-X100 in PBS for $3 \mathrm{~min}$, and mounted with DAPI. A minimum of 20 and up to 80 independent cells were analyzed for each condition and experiment, and quantification of the number of Alexa555-EGF structures per cell was performed by an observer blind to the conditions [31].

\subsection{Cell Extracts and Western Blotting}

HeLa cells were collected $48 \mathrm{~h}$ after transfection, washed in PBS, and resuspended in cell lysis buffer ( $1 \%$ SDS in PBS containing $1 \mathrm{mM} \mathrm{PMSF}, 1 \mathrm{mM} \mathrm{Na}_{3} \mathrm{VO}_{4}$, and $5 \mathrm{mM} \mathrm{NaF}$ ). Lysed extracts were sonicated, boiled and centrifuged at $13,500 \mathrm{rpm}$ for $10 \mathrm{~min}$ at $4{ }^{\circ} \mathrm{C}$, and the protein concentration of supernatants was estimated using the BCA assay (Pierce). Where indicated, HEK293T cells were transfected as previously described [31], and extracts were prepared as described above. Proteins were resolved by SDS-PAGE and electrophoretically transferred to nitrocellulose membranes. Membranes were blocked in blocking buffer (LiCOR Biosciences, Li-COR Odyssey PBS blocking buffer, Lincoln, NE, USA, 927-40000) for $1 \mathrm{~h}$ at room temperature, and incubated with primary antibodies in blocking buffer overnight at $4{ }^{\circ} \mathrm{C}$. Antibodies used for immunoblotting included rabbit polyclonal anti-GFP (1:2000; 
Abcam, Cambridge, UK, ab6556), mouse monoclonal anti-GAPDH (1:2000; Abcam, ab9484), mouse monoclonal anti-flag (1:500; Sigma, F1804), mouse monoclonal anti-tubulin (clone DM1A; 1:10,000; Sigma), rabbit monoclonal anti-RAB8A (1:1000; Abcam, ab188574), mouse monoclonal anti-RAB10 (1:1000; Sigma; SAB53000028), rabbit polyclonal anti-RAB7 (1:1000; Sigma, R4779), mouse monoclonal anti-RAB11 (1:1000; BD Biosciences, Madrid, Spain, 610656), rabbit monoclonal anti-RAB4 (1:1000; Abcam, ab109009), sheep polyclonal anti-RAB29 (1:250; S984D, MRC-PPU Reagents), and rabbit monoclonal anti-phosphoT73-RAB10 (1:1000; Abcam, ab230261). Secondary antibodies included goat anti-rabbit or anti-mouse IRDye $800 \mathrm{CW}$, and goat anti-rabbit or anti-mouse IRDye $680 \mathrm{CW}(1: 14,000)$, and blots were imaged via near infrared fluorescent detection using Odyssey CLx Imaging System, with quantification performed using the instrument's Image Studio software.

Alternatively, and in all cases employing antibodies raised in sheep, membranes were blocked in blocking buffer ( $5 \%$ milk in $0.1 \%$ Tween-20/TBS) for $8 \mathrm{~h}$, and primary antibodies diluted in blocking buffer and incubated overnight at $4{ }^{\circ} \mathrm{C}$. Membranes were washed three times in $0.1 \%$ Tween-20/TBS, followed by incubation with HRP-conjugated rabbit anti-sheep antibodies and detection using ECL reagents (Roche Diagnostic GmbH, Barcelona, Spain). A series of timed exposures were undertaken to ensure that densitometric analyses were performed at exposures within the linear range, and the films were scanned and analysed using Quantity One (Bio-Rad, Hercules, CA, USA).

\subsection{GST-RILP Pulldown Assays for Determination of Active RAB7}

GST-RILP pulldown assays were performed as previously described in detail [31,59]. In brief, freshly purified GST-RILP protein bound to GSH-Sepharose 4B beads (GE Healthcare) was incubated with extracts from transfected HEK293T cells (one $10 \mathrm{~cm}$ diameter dish per assay) in pulldown buffer (20 mM HEPES, pH 7.4, $100 \mathrm{mM} \mathrm{NaCl}, 5 \mathrm{mM} \mathrm{MgCl}_{2}, 1 \%$ Triton X-100, and $1 \mathrm{mM} \mathrm{PMSF}$ ), and incubated overnight in a rotary wheel at $4{ }^{\circ} \mathrm{C}$. Beads were washed twice with ice-cold pulldown buffer, and bound protein eluted with $40 \mu \mathrm{L}$ of $1 \times$ sample buffer/ $\beta$-mercaptoethanol and heating for $4 \mathrm{~min}$ at $95^{\circ} \mathrm{C}$ prior to SDS-PAGE.

\subsection{Statistical Analysis}

All data are expressed as means \pm S.E.M. One-way analysis of variance (ANOVA) with Tukey's post hoc test was employed, and significance was set at $p<0.05$. Significance values for all data are indicated in the figure legends, and all statistical analyses and graphs were performed using Prism software version 7.0 (GraphPad, San Diego, CA, USA).

\section{Results}

\subsection{G2019S LRRK2-Mediated Endolysosomal Trafficking Defects are Rescued by Active RAB10 and Mimicked} by Knockdown of RAB10

To determine whether RAB10 modulates the pathogenic LRRK2-mediated endolysosomal trafficking deficits, we used the EGFR trafficking assay [30,31]. Upon ligand binding using high concentrations of EGF, the EGFR is internalized by clathrin-mediated endocytosis and sorted to lysosomes for degradation [28]. The surface availability of the receptor can be determined by quantifying the binding of fluorescent EGF to cells at $4{ }^{\circ} \mathrm{C}$, and the endocytic trafficking and degradation by quantifying the amount of endocytosed fluorescent EGF at $37^{\circ} \mathrm{C}$ over time, respectively. HeLa cells were co-transfected with flag-tagged G2019S LRRK2 and either with GFP or with GFP-tagged RAB10 variants, and binding and degradation of fluorescently labelled EGF quantified (Figure 1A,B). As previously described [30,31], expression of flag-tagged G2019S LRRK2 reduced the binding of fluorescent EGF at $4{ }^{\circ} \mathrm{C}$, and impaired the clearance/degradation of internalized fluorescent EGF upon incubation of cells at $37^{\circ} \mathrm{C}$ (Figure 1A-D). GFP-tagged wildtype RAB10, or GTP-locked, constitutively active RAB10-Q68L were both localized to a tubular perinuclear compartment, while GDP-locked inactive RAB10-T23N was largely cytosolic (Figure S1A). Both wildtype RAB10 and RAB10-Q68L 
were expressed to similar degrees, and did not interfere with the co-expression of G2019S LRRK2 (Figure S1B). Expression of GFP-tagged RAB10 variants on their own was without effect on EGF binding or degradation (Figure 1E,F). However, when co-expressed with pathogenic G2019S LRRK2, active RAB10-Q68L fully rescued the decrease in EGF binding and the impairment in EGFR degradation, which was not observed with wildtype RAB10 or with the inactive RAB10 variant (Figure 1C,D), suggesting that pathogenic LRRK2 may cause the inactivation of RAB10.
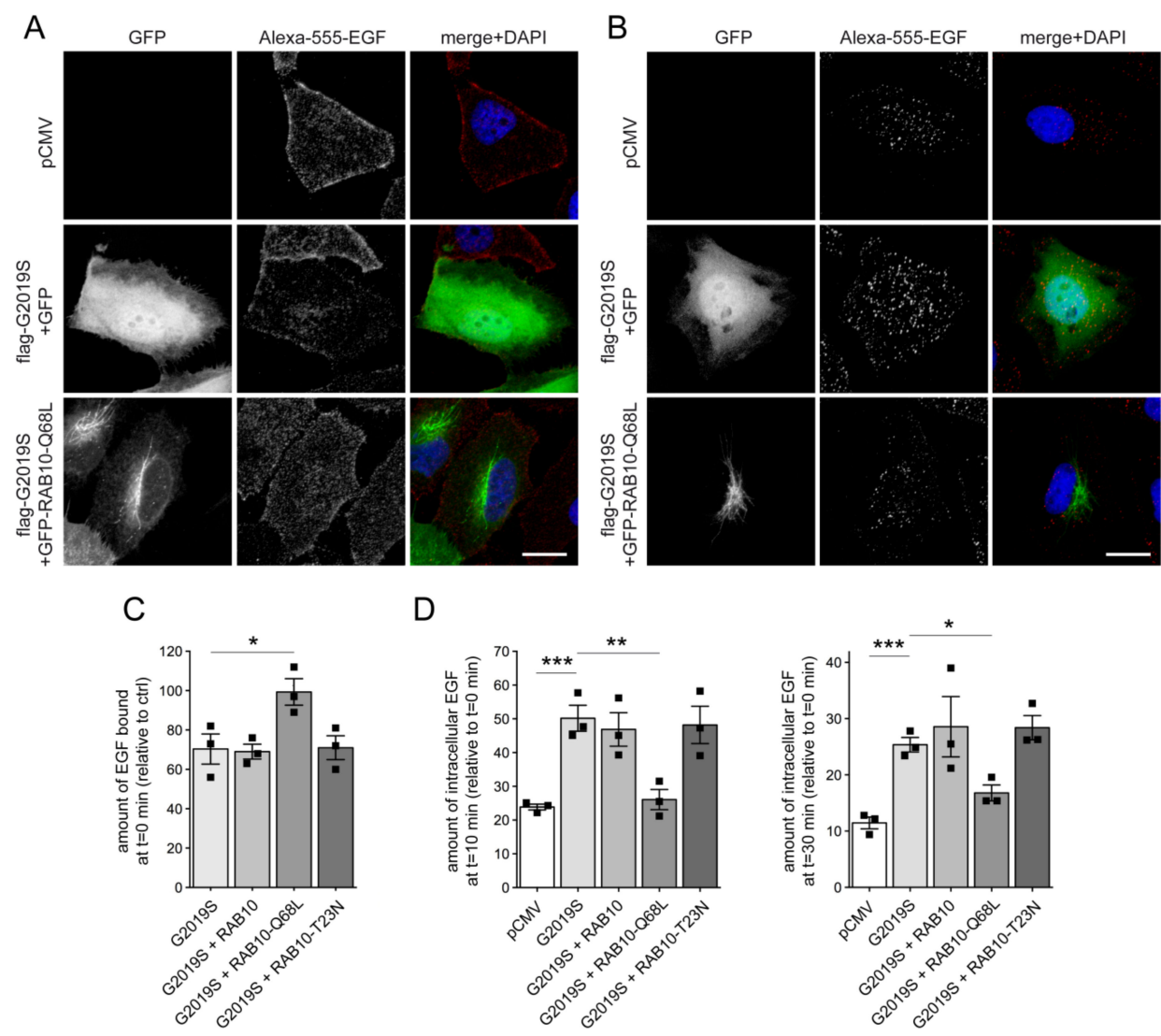

D
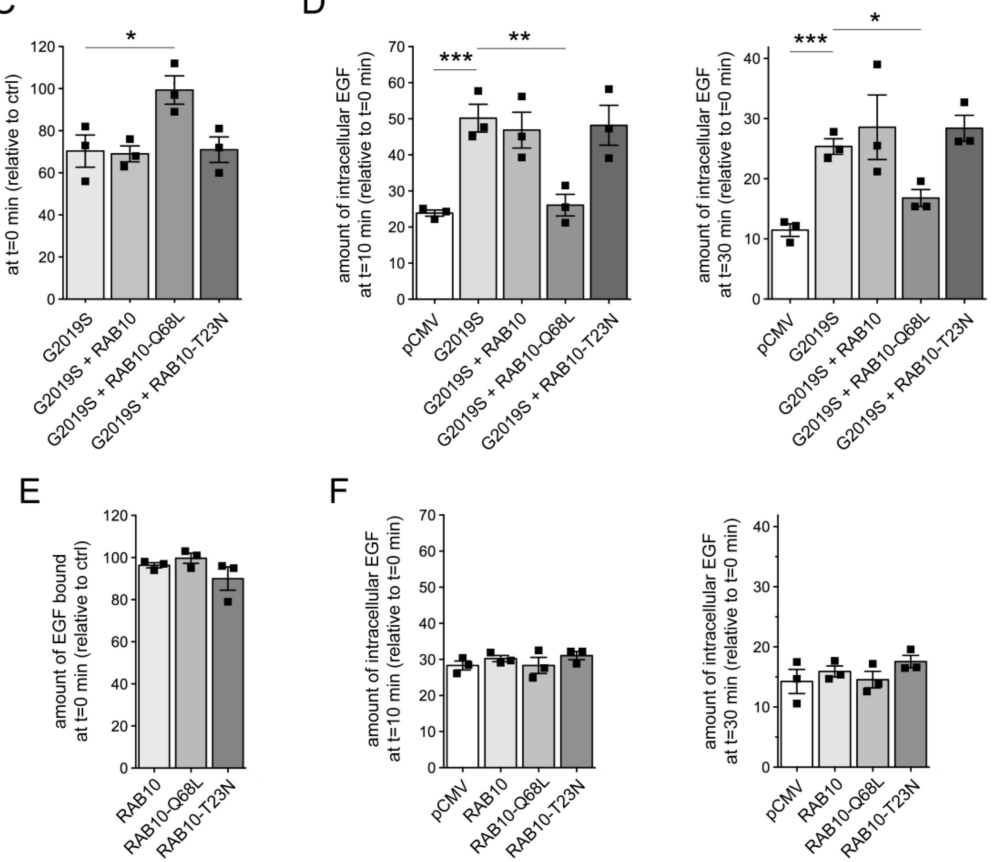

$\mathrm{F}$
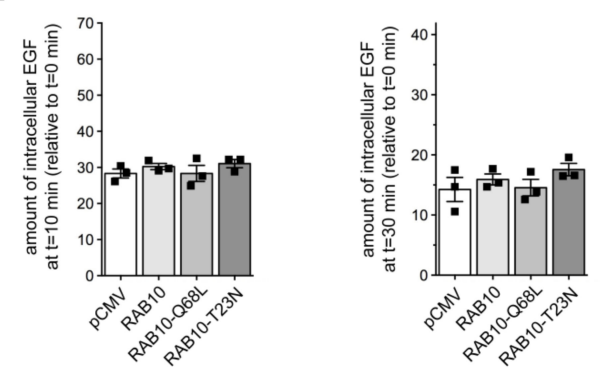

Figure 1. Active RAB10 rescues the G2019S leucine-rich repeat kinase 2 (LRRK2)-mediated deficit in epidermal growth factor (EGF) binding and degradation. (A) HeLa cells were transfected with either pCMV, or cotransfected with flag-tagged G2019S LRRK2 and GFP or GFP-tagged RAB10-Q68L as indicated. Cells were incubated with Alexa555-EGF for $20 \mathrm{~min}$ at $4{ }^{\circ} \mathrm{C}$, followed by washing to remove unbound fluorescent EGF before fixation $(\mathrm{t}=0 \mathrm{~min})$. Scale bar, $10 \mu \mathrm{m}$. (B) Same as in $(\mathbf{A})$, but upon incubation and washing, cells were shifted to $37^{\circ} \mathrm{C}$ for $10 \mathrm{~min}$ to allow for the internalization and degradation of fluorescent EGF. Scale bar, $10 \mu \mathrm{m}$. (C) Cells were co-transfected with G2019S LRRK2 and either GFP, or GFP-tagged RAB10 constructs as indicated, and the amount of surface-bound fluorescent EGF was quantified. $\mathrm{N}=3$ experiments; ${ }^{*} p<0.05$. (D) Cells were co-transfected as indicated, and the amount of internalized Alexa555-EGF in transfected cells was quantified after $10 \mathrm{~min}$ (left) and $30 \mathrm{~min}$ (right) of internalization, with values normalized to the amount of fluorescent EGF binding at $\mathrm{t}=0 . \mathrm{N}=3$ experiments; ${ }^{*} p<0.05 ;{ }^{* *} p<0.01$; ${ }^{* * *} p<0.005$. 
(E) The amount of surface-bound fluorescent EGF was quantified at $\mathrm{t}=0 \mathrm{~min}$ from cells transfected with the indicated GFP-tagged RAB10 constructs, and normalized to EGF surface binding of pCMV-transfected cells (ctrl). N=3 experiments. (F) The amount of fluorescent EGF was quantified after $10 \mathrm{~min}$ (left) and $30 \mathrm{~min}$ (right) upon internalization, and normalized to the amount of Alexa555-EGF binding for each condition at $\mathrm{t}=0 \mathrm{~min}$, thus reflecting the percentage of internalized bound fluorescent EGF. $\mathrm{N}=3$ experiments. All bars represent mean \pm s.e.m.

As another means to analyze the effect of RAB10 inactivation on EGF binding and EGFR trafficking, we performed siRNA experiments. Knockdown of RAB10 caused a pronounced decrease in RAB10 protein levels $48 \mathrm{~h}$ post-transfection (Figure 2A,B), while the steady-state levels of several other RAB proteins including RAB8A (Figure 2A,B) or RAB7A remained unchanged (Figure S2). The knockdown of RAB10 was accompanied by a significant decrease in EGF surface binding and EGFR degradation (Figure 2C,D). A siRNA-resistant version of RAB10, but not wildtype siRNA-sensitive RAB10, rescued the effect of RAB10 knockdown on EGF binding and EGFR trafficking (Figure 2E-G), indicating that the effects were owing to the specific knockdown of RAB10. Thus, siRNA of RAB10 mimics the endolysosomal trafficking deficits mediated by G2019S LRRK2 expression.

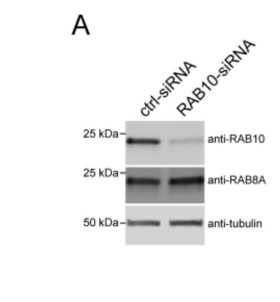

E

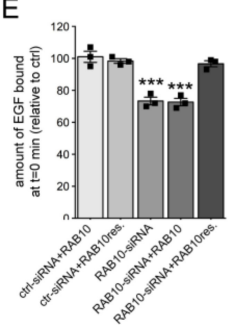

B

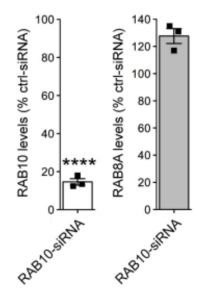

C

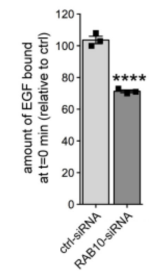

$\mathrm{F}$

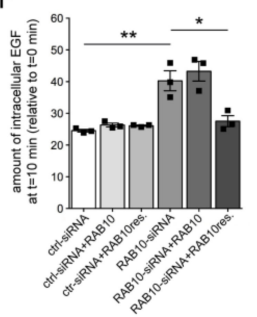

D
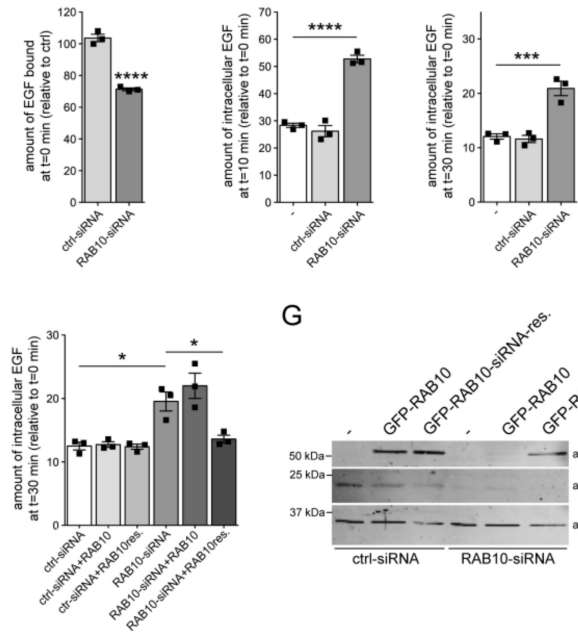

G

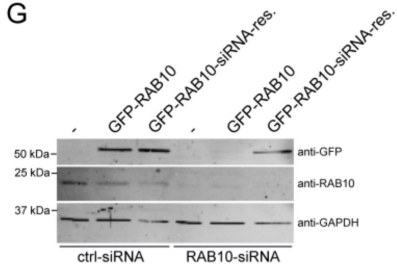

Figure 2. Knockdown of RAB10 mimics the EGF trafficking deficits observed upon G2019S LRRK2 expression. (A) HeLa cells were either transfected with ctrl-siRNA or RAB10-siRNA, and cell extracts were analyzed by Western blotting for RAB10 protein levels, RAB8A protein levels, or tubulin as loading control. (B) Quantification of RAB10 and RAB8A protein levels in the presence of RAB10-siRNA, normalized to the levels in the presence of ctrl-siRNA. Bars represent mean \pm s.e.m. $\left(\mathrm{N}=3\right.$ independent experiments; $\left.{ }^{* * * *} p<0.001\right)$. (C) Cells were either left untreated (-), or treated with ctrl-siRNA or RAB10-siRNA, and the amount of surface-bound fluorescent EGF was quantified. $\mathrm{N}=3$ independent experiments; ${ }^{* * *} p<0.001$. (D) Cells were either untreated (-), or treated with ctrl-siRNA or RAB10-siRNA, and internalized fluorescent EGF was quantified at $10 \mathrm{~min}$ (left) and 30 min (right). $\mathrm{N}=3$ independent experiments; ${ }^{* * *} p<0.005 ;{ }^{* * * *} p<0.001$. (E) Cells were either transfected with ctrl-siRNA or RAB10-siRNA in the absence or presence of wildtype or siRNA-resistant GFP-tagged RAB10 as indicated, and the amount of surface-bound fluorescent EGF was quantified. $\mathrm{N}=3$ independent experiments. ${ }^{* * *} p<0.005$. (F) Cells were either transfected with ctrl-siRNA or RAB10-siRNA in the absence or presence of wildtype or siRNA-resistant GFP-RAB10, and internalized fluorescent Alexa555-EGF quantified upon $10 \mathrm{~min}$ (left) or $30 \mathrm{~min}$ (right) of internalization. $\mathrm{N}=3$ independent experiments. ${ }^{*} p<0.05 ;{ }^{* *} p<0.01$. (G) Cells were either transfected with ctrl-siRNA or RAB10-siRNA, and with either wildtype or siRNA-resistant GFP-tagged RAB10 as indicated, and cell extracts $(30 \mu \mathrm{g})$ analyzed by Western blotting for GFP-RAB10 levels, endogenous RAB10 levels, and GAPDH as loading control. 


\subsection{Knockdown of RAB10 Causes a Decrease in RAB7 Activity and Mistargeting of EGF into an RAB4 Compartment}

We previously reported that knockdown of RAB8A mimicked the effects of G2019S LRRK2 on endolysosomal trafficking by decreasing the activity of RAB7 [31], a crucial regulator of endolysosomal trafficking pathways $[60,61]$. The decrease in RAB7 activity upon either G2019S LRRK2 expression or knockdown of RAB8A was associated with the accumulation of EGF in a RAB4-positive endocytic compartment, and all trafficking deficits were rescued upon expression of active RAB7A [31]. Similarly, the deficits in EGF surface binding and EGFR degradation induced upon siRNA of RAB10 were rescued when overexpressing active, GTP-locked RAB7A (RAB7A-Q67L), but not wildtype or GDP-locked RAB7A (RAB7A-T22N) (Figure 3A,B), even though all RAB7A versions were expressed to comparable degrees (Figure $3 C$ ). In addition, knockdown of RAB10 was associated with the redistribution of EGF into a vesicular recycling compartment colocalizing with RAB4, which was rescued upon active RAB7A expression (Figure S3).

A

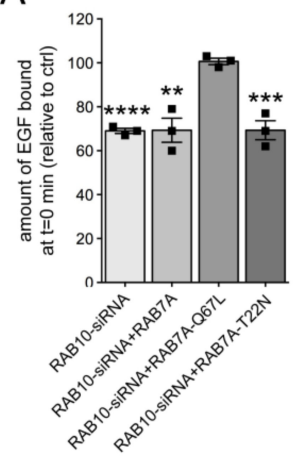

C

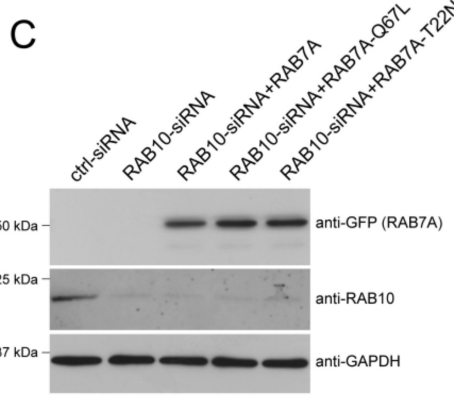

B
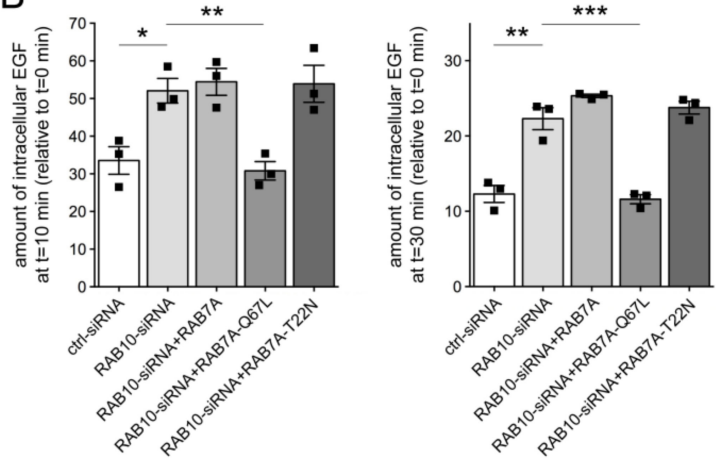

D

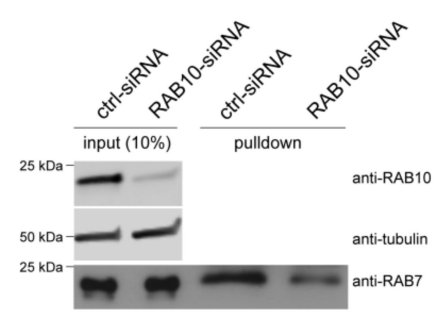

$E$

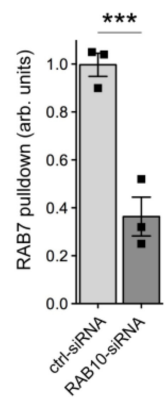

Figure 3. Knockdown of RAB10 decreases RAB7 activity. (A) HeLa cells were either transfected with ctrl-siRNA, or with RAB10-siRNA in the absence or presence of GFP-tagged RAB7A constructs as indicated, and surface-bound fluorescent EGF was quantified. $\mathrm{N}=3$ independent experiments. ${ }^{* *} p<0.01$; ${ }^{* * *} p<0.005 ; * * * * p<0.001$. (B) Cells were either transfected with ctrl-siRNA, or with RAB10-siRNA in the absence or presence of GFP-tagged RAB7A constructs as indicated, and internalized fluorescent EGF was quantified at $10 \mathrm{~min}$ (left) and $30 \mathrm{~min}$ (right) upon internalization. $\mathrm{N}=3$ independent experiments. ${ }^{*} p<0.05$; ${ }^{* *} p<0.01$; ${ }^{* *} p<0.005$. (C) Cells were treated with ctrl-siRNA or RAB10-siRNA, and transfected with the indicated RAB7A constructs, and cell extracts $(30 \mu \mathrm{g})$ were analyzed by Western blotting for GFP-RAB7A protein levels, endogenous RAB10 protein levels, and GAPDH as loading control. (D) Cells were either treated with ctrl-siRNA or RAB10-siRNA. The RAB7-binding domain of RILP coupled to GST was used to pull down the GTP-bound form of RAB7 from cell lysates $(300 \mu \mathrm{g})$, and 10\% of input was run alongside pulldowns to show equal levels of total RAB7 protein in ctrl-siRNA or RAB10-siRNA-treated cells. The levels of RAB10 and tubulin were analyzed on a separate gel. (E) Quantification of the type of experiments is depicted in (D), with the amount of RAB7 isolated by GST-RILP expressed relative to input. $\mathrm{N}=3$ independent experiments. ${ }^{* * *} p<0.005$. 
To gain direct evidence for a decrease in RAB7A activity upon siRNA of RAB10, we performed effector pulldown assays. Cells were treated with either control siRNA or with RAB10-siRNA, and cell lysates were subjected to pulldowns with the RAB7-binding domain of RILP to selectively isolate active, GTP-bound RAB7 [31,59]. Studies of this type further indicate that the fraction of endogenous active RAB7 was significantly reduced upon RAB10-siRNA (Figure 3D,E).

Because either knockdown of RAB8A or of RAB10 phenocopied the effects of pathogenic G2019S LRRK2 expression, we wondered whether these RAB proteins may act in a functionally redundant manner with respect to EGF trafficking and EGFR degradation. Indeed, the decrease in EGF binding and EGFR degradation mediated by knockdown of RAB10 was rescued upon expression of active, but not wildtype or inactive RAB8A (Figure 4A,B). Conversely, the deficits in EGF binding and EGFR degradation upon siRNA of RAB8A were rescued upon expression of active, but not wildtype or inactive RAB10 (Figure 4C,D). Thus, impairing the function of either RAB8A or RAB10 causes endolysosomal trafficking deficits identical to G2019S LRRK2 expression, which are associated with a decrease in RAB7 activity and culminate in the accumulation of EGF in a non-degradative, RAB4-positive recycling compartment.

A

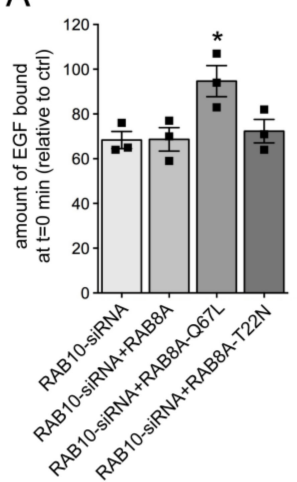

C

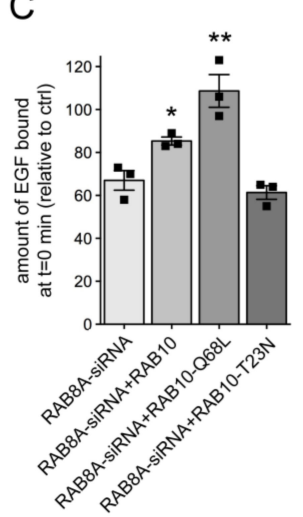

B
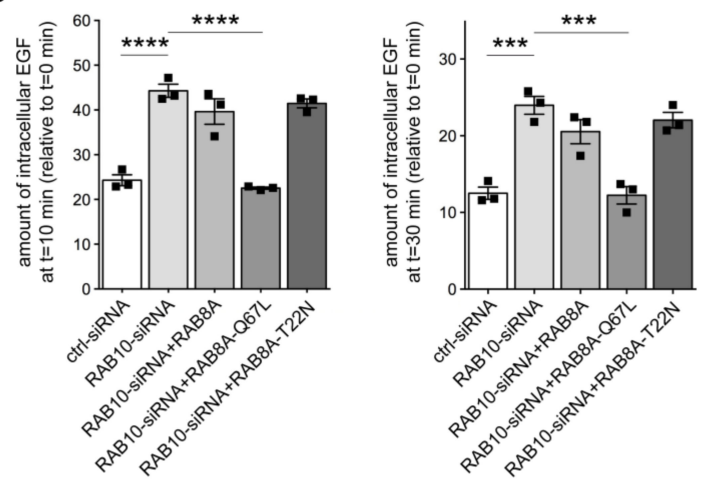

D
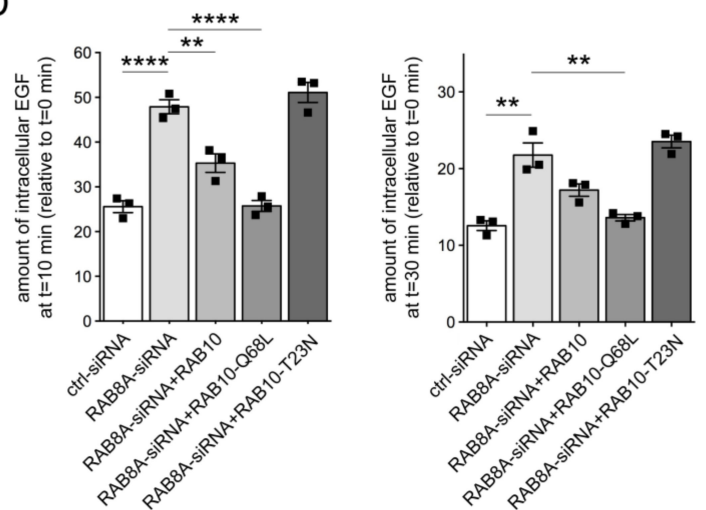

Figure 4. RAB8A and RAB10 are functionally redundant in regulating endolysosomal EGF trafficking. (A) HeLa cells were either transfected with ctrl-siRNA or RAB10-siRNA, and co-transfected with GFP-tagged RAB8A constructs as indicated, and the amount of surface-bound fluorescent EGF was quantified. $\mathrm{N}=3$ independent experiments; ${ }^{*} p<0.05$. (B) Cells were transfected with ctrl-siRNA or RAB10-siRNA, and co-transfected with GFP-tagged RAB8A constructs as indicated, and internalized fluorescent EGF was quantified at $10 \mathrm{~min}$ (left) and $30 \mathrm{~min}$ (right). $\mathrm{N}=3$ independent experiments; ${ }^{* * *} p<0.005 ;{ }^{* * *} p<0.001$. (C) HeLa cells were either transfected with ctrl-siRNA or RAB8A-siRNA, and co-transfected with GFP-tagged RAB10 constructs as indicated, and the amount of surface-bound fluorescent EGF was quantified. $\mathrm{N}=3$ independent experiments; ${ }^{*} p<0.05 ;{ }^{* *} p<0.01$. (D) Cells were transfected with ctrl-siRNA or RAB8A-siRNA, and co-transfected with GFP-tagged RAB10 constructs as indicated, and internalized fluorescent EGF was quantified at $10 \mathrm{~min}$ (left) and $30 \mathrm{~min}$ (right). $\mathrm{N}=3$ independent experiments; ${ }^{* *} p<0.01 ; * * * * 0.001$. 


\subsection{G2019S LRRK2-Mediated Endolysosomal Trafficking Defects are Rescued by RAB29 Expression}

Previous studies indicate that overexpression of RAB29 causes recruitment of LRRK2 to the trans-Golgi network (TGN), which in turn activates LRRK2 as assessed by RAB10 phosphorylation [23,54-56]. Surprisingly, while GFP-tagged RAB29 largely colocalized with a Golgi marker, coexpression of RAB29 with flag-tagged G2019S LRRK2 revealed that this did not result in the efficient recruitment of LRRK2 to the Golgi complex (Figure 5A). We next assessed the effect of RAB29 expression on the pathogenic LRRK2-mediated endolysosomal trafficking deficits. When coexpressed with G2019S LRRK2, RAB29 rescued the LRRK2-mediated decrease in EGF binding and the impairment in EGFR degradation (Figure 5B,C). In contrast, two mutant RAB29 versions previously described to cause RAB29 inactivation $[23,54,55]$ did not rescue the LRRK2-mediated endolysosomal trafficking deficits (Figure 5B,C). Furthermore, expression of the various RAB29 constructs on their own was without effect (Figure 5D,E), even though the RAB29 variants were expressed to similar degrees and did not change expression levels of G2019S LRRK2 (Figure 5F). Finally, wildtype, but not mutant RAB29 versions were also found to rescue the G2019S LRRK2-mediated accumulation of EGF in an RAB4-positive endocytic compartment (Figure S4A).

We reasoned that the previously reported RAB29-mediated recruitment and activation of LRRK2 may be dependent on overexpression levels. Indeed, when employing another transfection reagent to achieve higher expression levels, GFP-RAB29 expression caused a pronounced recruitment of G2019S LRRK2 (Figure S5). Under these conditions, while expression of RAB29 variants on their own still was without effect (Figure S6A,B), co-expression of wildtype RAB29 with G2019S LRRK2 failed to rescue the LRRK2-mediated deficits in EGF binding and EGFR degradation (Figure S6C,D), in contrast to what was observed with low-level RAB29 expression in parallel experiments (Figure S6E,F). Thus, GFP-RAB29 is largely localized to the Golgi complex independent of expression levels. However, and at least as analyzed here, the RAB29-mediated recruitment of LRRK2 to the Golgi complex is only evident upon higher expression levels, and is associated with an impairment of the RAB29-mediated rescue of the endolysosomal trafficking deficits owing to G2019S LRRK2 expression.

\subsection{RAB29 Expression Rescues the Endolysosomal Trafficking Deficits Mediated by G2019S LRRK2 or Knockdown of Either RAB8A or RAB10}

We next assessed whether the LRRK2-mediated endolysosomal trafficking deficits may be owing to RAB29 inactivation. Knockdown of RAB29 was without effect on EGF binding and EGFR degradation (Figure S7A-D), in contrast to what we observed with knockdown of RAB8A [31] or RAB10 (Figure 2). Furthermore, transient disruption of the Golgi complex by brefeldin A (BFA) treatment did not alter EGF binding or EGFR trafficking per se (Figure S7E-G), suggesting that the LRRK2-mediated endolysosomal trafficking deficits are neither mimicked by RAB29 inactivation nor dependent on Golgi integrity. Importantly, while BFA treatment caused the perinuclear dispersal of a Golgi marker as well as of GFP-RAB29 (Figure 6A,B), it did not interfere with the RAB29-mediated rescue of the deficits in EGF binding and EGFR degradation owing to the presence of G2019S LRRK2 (Figure 6C,D). Thus, the RAB29-mediated rescue of the trafficking deficits owing to G2019S LRRK2 expression does not require an intact Golgi complex. 


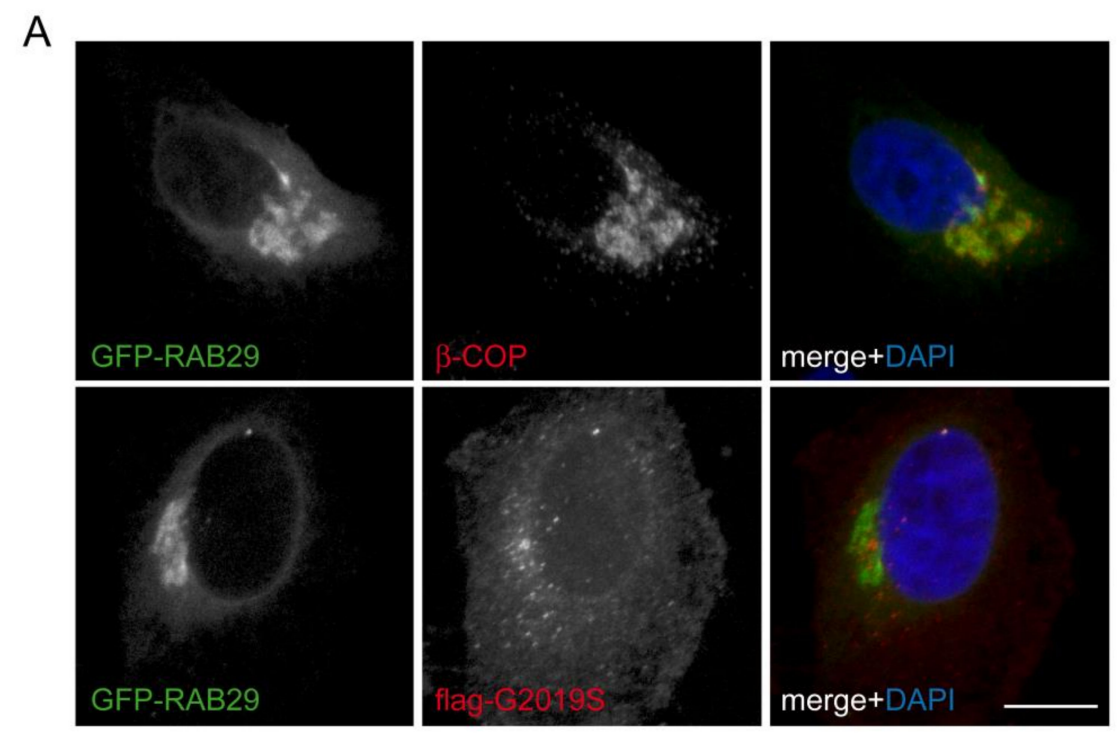

B

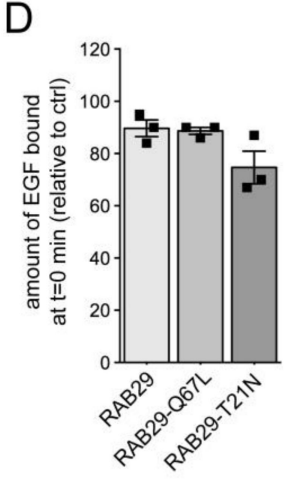

C

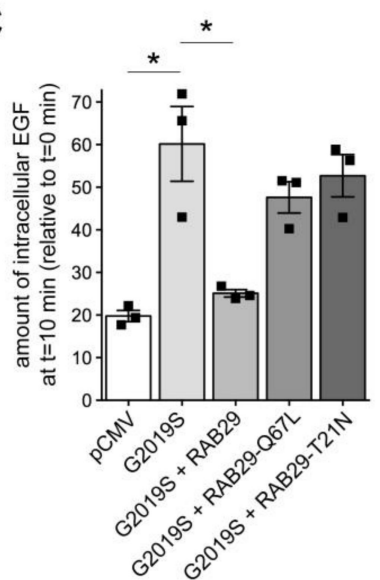

E

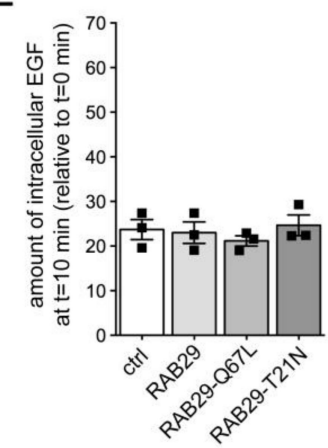

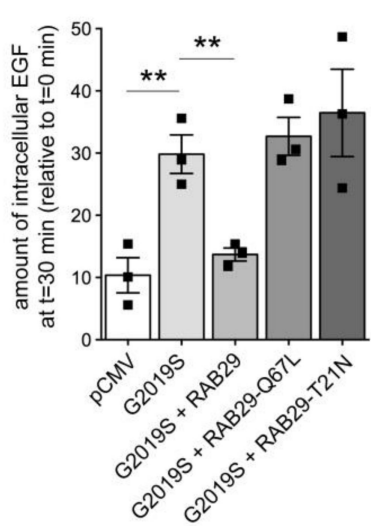

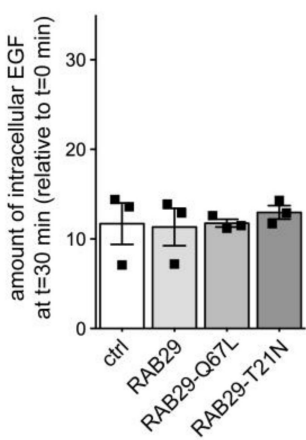

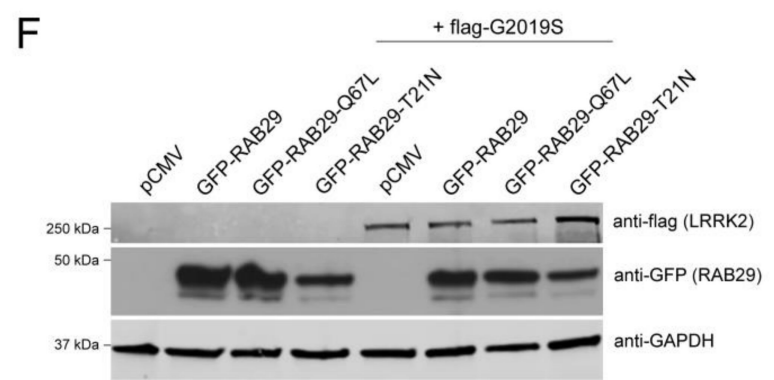

Figure 5. RAB29 rescues the G2019S LRRK2-mediated EGF trafficking deficits. (A) Top: Example of HeLa cell transfected with GFP-tagged RAB29 using Lipofectamine 2000 (LF2000), and stained with Golgi 
marker ( $\beta$-COP) and DAPI. Bottom: Example of HeLa cell co-transfected with GFP-tagged RAB29 and flag-tagged G2019S LRRK2 using LF2000, and stained with flag antibody and DAPI. Scale bar, $10 \mu \mathrm{m}$. (B) Cells were transfected with either pCMV, or cotransfected with flag-tagged G2019S LRRK2 and GFP or GFP-tagged RAB29 constructs as indicated, and surface-bound fluorescent EGF quantified. $\mathrm{N}=3$ independent experiments. ${ }^{*} p<0.05$. (C) Cells were transfected with either pCMV, or cotransfected with flag-tagged G2019S LRRK2 and GFP or GFP-tagged RAB29 constructs, and internalized fluorescent EGF was quantified at $10 \mathrm{~min}$ (left) and $30 \mathrm{~min}$ (right) upon internalization. $\mathrm{N}=3$ independent experiments. ${ }^{*} p<0.05 ;{ }^{* *} p<0.01$. (D) HeLa cells were transfected with the indicated GFP-tagged RAB29 constructs using Lipofectamine 2000 (LF2000), and surface-bound fluorescent EGF quantified. $\mathrm{N}=3$ independent experiments. (E) Cells were transfected with the indicated GFP-tagged RAB29 constructs, and internalized fluorescent EGF was quantified at $10 \mathrm{~min}$ (left) and $30 \mathrm{~min}$ (right) upon internalization. $\mathrm{N}=3$ independent experiments. (F) HeLa cells were transfected with empty pCMV vector, with GFP-tagged RAB29 constructs, or with flag-G2019S LRRK2 along with GFP-tagged RAB29 constructs as indicated, and cell extracts $(30 \mu \mathrm{g})$ analyzed by Western blotting for flag, GFP, and GAPDH as loading control.

A

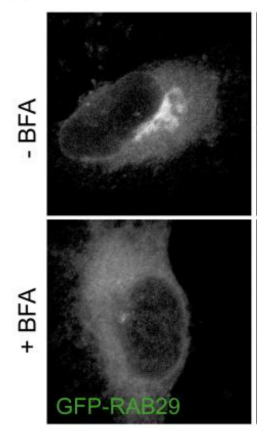

C

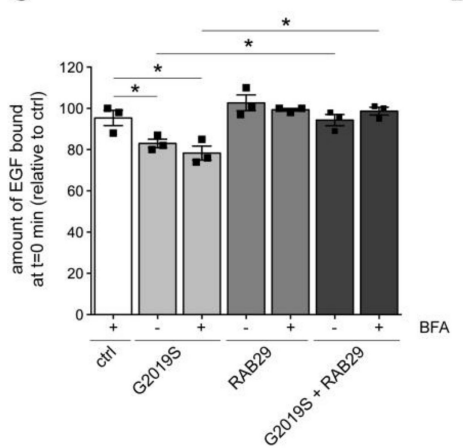

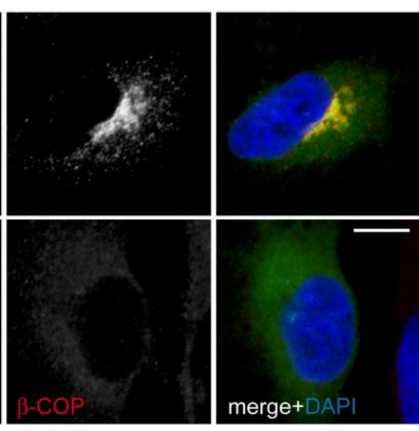

B

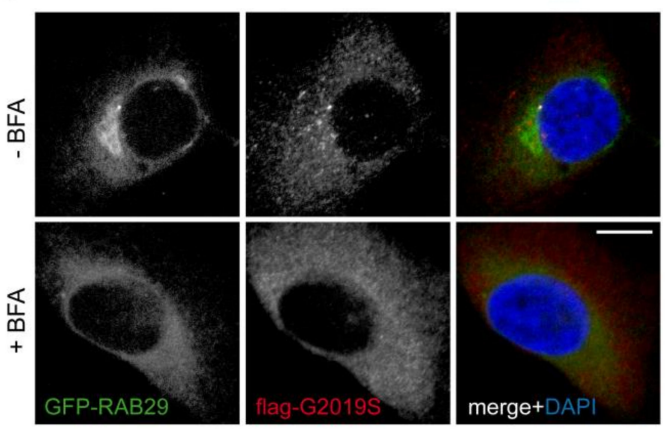

$D$

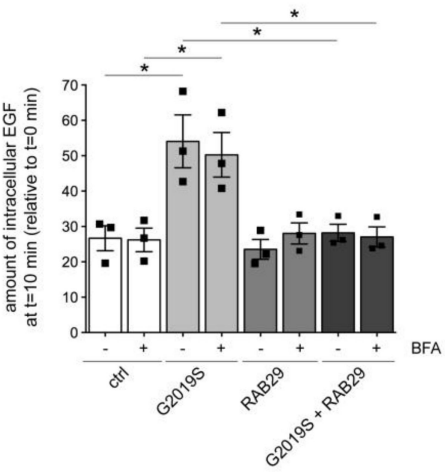

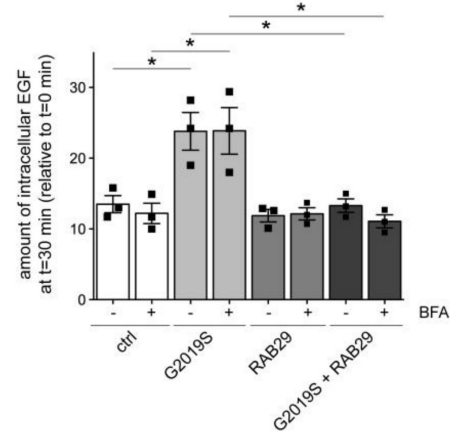

Figure 6. The G2019S LRRK2-mediated deficit in EGF trafficking is rescued by RAB29 independent of Golgi integrity. (A) Example of HeLa cell transfected with GFP-RAB29 using Lipofectamine 2000 (LF2000), and stained for $\beta$-COP and DAPI in either the absence or presence of BFA treatment $(5 \mu \mathrm{g} / \mathrm{mL}$, $2 \mathrm{~h}$ ) as indicated. Scale bar, $10 \mu \mathrm{m}$. (B) Example of HeLa cell cotransfected with GFP-RAB29 and flag-tagged G2019S LRRK2 using LF2000, and stained for flag and DAPI in either the presence or absence of BFA treatment $(5 \mu \mathrm{g} / \mathrm{mL}, 2 \mathrm{~h})$ as indicated. Scale bar, $10 \mu \mathrm{m}$. (C) HeLa cells were either transfected with pCMV (ctrl), flag-tagged G2019S LRRK2, GFP-tagged RAB29, or cotransfected with flag-tagged G2019S LRRK2 and GFP-tagged RAB29 using LF2000 as indicated, and either treated with or without BFA $(5 \mu \mathrm{g} / \mathrm{mL}, 2 \mathrm{~h})$ before determination of the amount of surface-bound fluorescent EGF. $N=3$ independent experiments; ${ }^{*} p<0.05$. (D) HeLa cells were either left untreated (ctrl), transfected with flag-tagged G2019S LRRK2, GFP-tagged RAB29, or cotransfected with flag-tagged G2019S LRRK2 and GFP-tagged RAB29 using LF2000, either treated with or without BFA ( $5 \mu \mathrm{g} / \mathrm{mL}, 2 \mathrm{~h}$ ) as indicated, and internalized fluorescent EGF was quantified at $10 \mathrm{~min}$ (left) and $30 \mathrm{~min}$ (right) upon internalization. $\mathrm{N}=3$ independent experiments. ${ }^{*} p<0.05$. 
We reasoned that RAB29 may play additional roles in membrane trafficking apart from those described at the Golgi complex, possibly overlapping with those of either RAB8A and/or RAB10. Indeed, expression of wildtype, but not inactive RAB29 variants rescued the deficits in EGF binding and EGFR degradation induced upon knockdown of either RAB10 (Figure 7A-C) or RAB8A (Figure 7D-F), with the RAB29 variants expressed to similar degrees (Figure 7C,F). Expression of wildtype RAB29 also reverted the accumulation of EGF in a RAB4-positive endocytic compartment induced upon knockdown of either RAB10 or RAB8A (Figure S4B,C). Finally, the trafficking deficits mediated by expression of a dominant-negative RAB7A mutant were rescued by RAB29 expression (Figure S8A-C), as previously described for active RAB8A [31]. These data indicate that RAB29 can rescue the endolysosomal trafficking deficits induced by either G2019S LRRK2 expression or by knockdown of RAB8A or RAB10 in a manner independent of its localization/function at the Golgi complex.

A

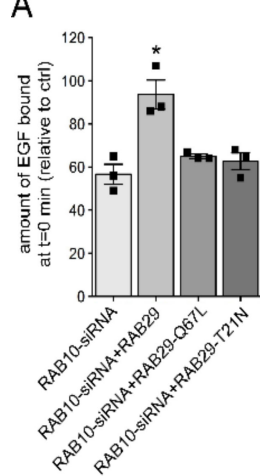

D

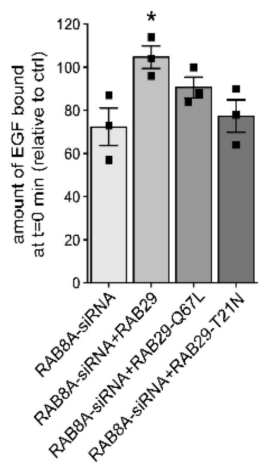

B

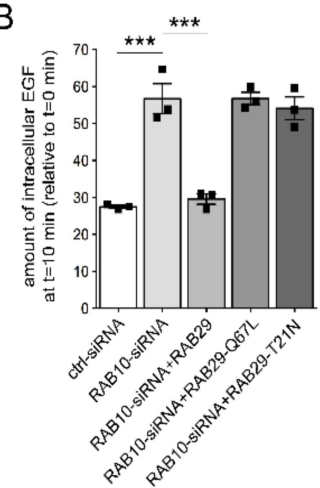

E

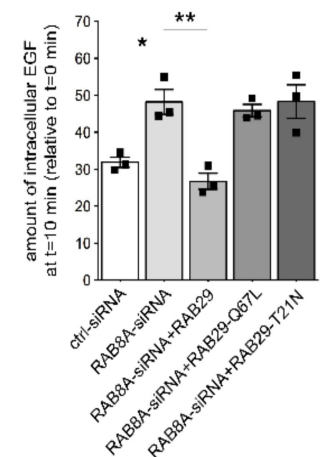

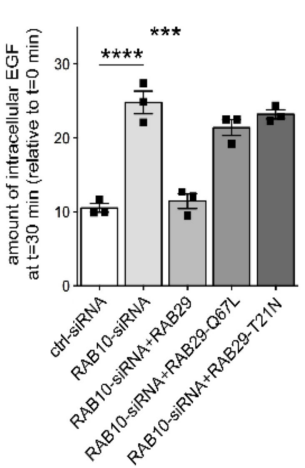

C

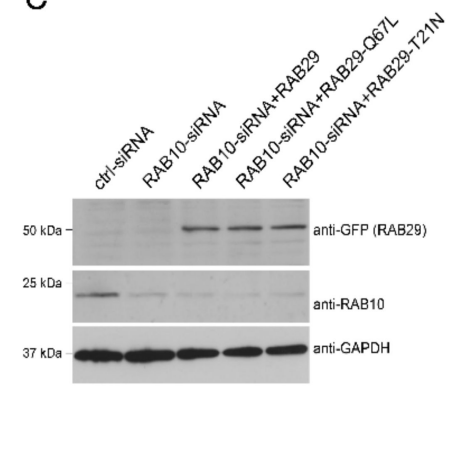

$\mathrm{F}$

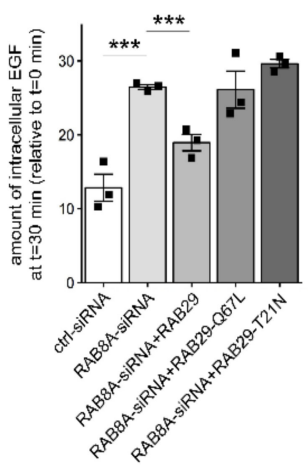

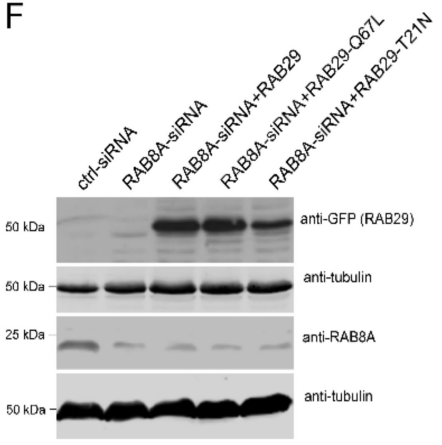

Figure 7. Deficits in EGF trafficking due to knockdown of RAB8A or RAB10 are rescued upon RAB29 expression. (A) HeLa cells were either transfected with ctrl-siRNA or RAB10-siRNA, and cotransfected with GFP-tagged RAB29 constructs as indicated, and the amount of surface-bound fluorescent EGF quantified. $N=3$ independent experiments; ${ }^{*} p<0.05$. (B) Cells were transfected with ctrl-siRNA or RAB10-siRNA, and cotransfected with GFP-tagged RAB29 constructs as indicated, and internalized fluorescent EGF was quantified at $10 \mathrm{~min}$ (left) and $30 \mathrm{~min}$ (right). $\mathrm{N}=3$ independent experiments; ${ }^{* * *} p<0.005 ;{ }^{* * * *} p<0.001$. (C) Cells were either transfected with ctrl-siRNA or RAB10-siRNA, and transfected with GFP-tagged RAB29 constructs as indicated, and cell extracts ( $30 \mu \mathrm{g})$ were analyzed by Western blotting for GFP-RAB29 levels, endogenous RAB10 levels, and GAPDH as loading control. (D) HeLa cells were either transfected with ctrl-siRNA or RAB8A-siRNA, and cotransfected with GFP-tagged RAB29 constructs as indicated, and the amount of surface-bound fluorescent EGF was quantified. $N=3$ independent experiments; ${ }^{*} p<0.05$. (E) Cells were transfected with ctrl-siRNA or RAB8A-siRNA, and cotransfected with GFP-tagged RAB29 constructs as indicated, and internalized fluorescent EGF was quantified at $10 \mathrm{~min}$ (left) and $30 \mathrm{~min}$ (right). $\mathrm{N}=3$ independent experiments; ${ }^{*} p<0.05 ;{ }^{* *} p<0.01 ;{ }^{* * *} p<0.005$. (F) Cells were either transfected with ctrl-siRNA or RAB8A-siRNA, and transfected with GFP-tagged RAB29 constructs as indicated, and cell extracts ( $30 \mu \mathrm{g})$ were analyzed by Western blotting for GFP-RAB29 levels, endogenous RAB8A levels, and tubulin as loading control. 


\section{Discussion}

In the present study, we find that the endolysosomal deficits mediated by pathogenic LRRK2 are mimicked by knockdown of RAB10 and rescued by expression of active RAB10. The deficits in endolysosomal trafficking/degradation of the EGFR by RAB10 inactivation are accompanied by the accumulation of the receptor in a RAB4-positive recycling compartment, and by a decrease in the levels of active, GTP-bound RAB7A, identical to what we previously described for pathogenic LRRK2 expression or knockdown of RAB8A [30,31]. In addition, the endolysosomal trafficking deficits mediated by knockdown of RAB10 are rescued by expression of active RAB8A and vice versa. These data are consistent with a model whereby the LRRK2-mediated deficits in endolysosomal trafficking are the result of a loss-of-function phenotype of either RAB10 or RAB8A, likely triggered by the phosphorylation and concomitant inactivation of these RAB proteins. Furthermore, our data indicate that RAB8A and RAB10 play functionally redundant roles, at least for the endocytic trafficking/degradation of the EGFR, as analyzed here. This is consistent with their colocalization to a perinuclear tubular endocytic recycling compartment [37-40], and with previous reports showing that RAB8 and RAB10 can cooperatively regulate other vesicular trafficking pathways including basolateral sorting, neurite outgrowth, and ciliogenesis [42-45]. Thus, the sum of phospho-RAB8 and phospho-RAB10 may be driving the pathogenic LRRK2-mediated vesicular trafficking deficits, and in the future, it will be important to determine the combined levels of both phospho-proteins, and whether LRRK2-mediated endolysosomal trafficking alterations occur in a manner dependent on RAB8A and RAB10 in dopaminergic neurons or other disease-relevant cell types. In addition, endocytic trafficking deficits have also been described for the dopamine D1 receptor and the transferrin receptor [62,63], and it will be interesting to determine whether they are owing to the same LRRK2-mediated inactivation of RAB8A and RAB10 as described here.

The accumulation of EGF in a RAB4-positive recycling compartment upon pathogenic LRRK2 expression or RNAi of either RAB8A or RAB10 reflects a crosstalk between recycling and degradative trafficking pathways, which has been described before [64-66]. For example, expression of a dominant-negative RAB4 construct alters both endocytic recyling and degradation events [64], and interfering with the integrity/function of the RAB8-positive early recycling compartment causes impaired degradation of the EGFR, which consequently accumulates in a RAB4-positive recycling compartment [65]. Thus, impairing the proper functioning of the RAB8/RAB10-positive perinuclear endocytic recycling compartment is associated with deficits in the complex crosstalk between recycling and degradative vesicular trafficking pathways, with various and potentially disease-relevant cellular outcomes.

The trafficking alterations mediated by pathogenic LRRK2 or knockdown of either RAB8A or RAB10 all converge on decreasing the amounts of GTP-bound, active RAB7. RAB7A plays a key role in the endolysosomal system by regulating trafficking from the late endosome/multivesicular body to the lysosome, and is also implicated in the process of lysosome reformation $[60,61,67,68]$. RAB7A does not serve as a substrate for the LRRK2 kinase activity in vitro or in intact cells [12,18,31], indicating that the decrease in RAB7A activity mediated by pathogenic LRRK2 expression occurs by indirect means. The specificity of intracellular vesicular trafficking pathways is often ensured by a so-called $\mathrm{RAB}$ cascade, whereby the effector of the upstream RAB protein acts as an activator (GEF) for the downstream RAB protein [69-72]. Such a RAB cascade has been described for the trafficking between RAB5-positive early endosomes and RAB7-positive late endosomes, whereby RAB5 is necessary and sufficient to drive GEF-dependent activation of RAB7 [73]. In the future, it will be important to determine whether a similar RAB cascade operates between RAB8/RAB10-positive and RAB7-positive compartments, which may underlie the observed decrease in RAB7 activity due to RAB8/RAB10 phosphorylation/inactivation.

In contrast to RAB8 and RAB10, knockdown of RAB29 did not cause endolysosomal trafficking deficits of the EGFR, suggesting that, under normal conditions, RAB29 is not required for this process. However, knockdown or knockout of RAB29 causes deficits in retrograde sorting events between the 
late endosome and the Golgi complex, which is crucial for the correct trafficking of lysosomal enzymes and associated with defects in lysosomal homeostasis $[22,27,74]$. Deficits in the trafficking/degradation of the EGFR may not have been evident owing to the incomplete and/or transient nature of the RAB29 knockdown, because alterations in the correct processing of lysosomal hydrolases have only been described in RAB29 knockout contexts, and may thus only arise as a consequence of the prolonged missorting of cargo [27,74].

While knockdown of RAB29 was without effect on EGFR trafficking, RAB29 expression rescued the pathogenic LRRK2-mediated endolysosomal trafficking deficits. This finding is consistent with previous studies reporting a beneficial effect of increasing RAB29 protein levels on Golgi-lysosome sorting events, neurite outgrowth, and dopaminergic cell survival in Drosophila neurons, and with transcriptome analysis suggesting that RAB29 risk variants correlate with a decrease in RAB29 expression levels [22]. As other studies have suggested that increased PD risk may be associated with increased RAB29 levels [23], further studies using newly generated antibodies specific against RAB29 are warranted to correlate PD risk with possible alterations in RAB29 protein levels.

The rescue of the LRRK2-mediated deficits upon RAB29 expression occurred in a manner largely independent of Golgi integrity, suggesting that RAB29 may play role(s) in membrane trafficking in addition to those described at the Golgi complex. Indeed, previous studies indicate that RAB29 colocalizes and interacts with RAB8, and regulates receptor recycling in T cells as well as the trafficking of endosome-derived ciliary cargo at the base of the cilium [75]. As RAB29 expression can functionally complement for the knockdown of either RAB8A or RAB10, it is tempting to speculate that at least a fraction of RAB29 may be localized to the recycling compartment, where it may be able to perform functions overlapping with those of RAB8A and/or RAB10 (Figure 8). Finally, our data show that the reported recruitment of LRRK2 to the Golgi complex by RAB29 [23,54-56] is only observed when RAB29 is overexpressed to a larger extent. This is consistent with findings indicating that most endogenous LRRK2 is not present on the Golgi complex, and that phospho-RAB8A/RAB10 is not trapped on the Golgi complex under normal conditions either $[39,55,57]$. While further work is required to understand the link between RAB29, LRRK2, and the etiology of PD, the present data highlight the possibility that, under more physiological conditions, RAB29 may be a positive regulator of non-Golgi-related membrane trafficking events impaired by pathogenic LRRK2, rather than controlling the localization and activation of LRRK2 at the Golgi complex.

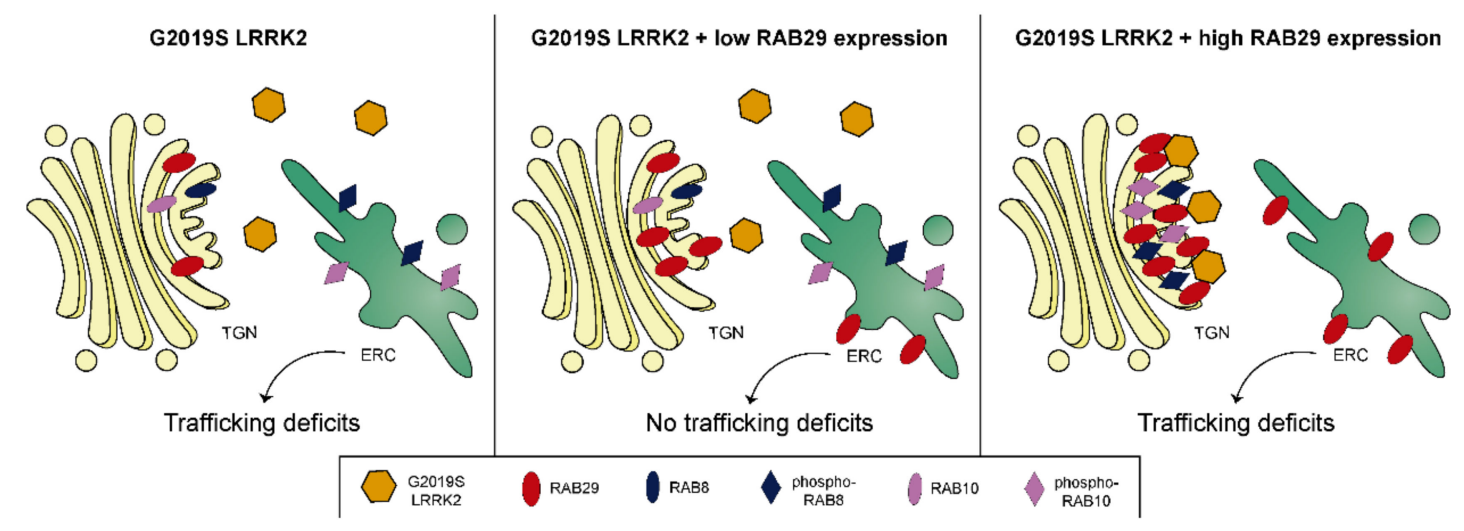

Figure 8. Model for the RAB29-mediated regulation of G2019S LRRK2 localization and rescue of trafficking deficits. Schematics summarizing the RAB29-mediated modulation of trafficking defects owing to pathogenic LRRK2. Left: RAB8A and RAB10 are mainly localized to the early recycling compartment (ERC), while endogenous RAB29 is mainly localized to the Golgi complex. Pathogenic G2019S LRRK2 causes phosphorylation (and thus inactivation) of RAB8A/RAB10 at the ERC, resulting in deficits of the endolysosomal trafficking of the EGFR (trafficking deficits). Middle: upon low/moderate 
overexpression of RAB29, G2019S LRRK2 remains cytosolic, while RAB29 localizes to the Golgi (TGN) and possibly also the ERC. G2019S LRRK2 causes phosphorylation/inactivation of RAB8A/RAB10 at the ERC, but non-Golgi-localized RAB29 is able to rescue the pathogenic LRRK2-mediated trafficking deficits, perhaps by positively regulating the remaining non-phosphorylated RAB8A/RAB10 at the ERC. Right: upon high overexpression of RAB29, G2019S LRRK2 is recruited to the Golgi complex where it phosphorylates and "traps" RAB8A/RAB10, resulting in a depletion of non-phosphorylated RAB8A/10 at the ERC. Under these conditions, non-Golgi-localized RAB29 is no longer able to rescue the pathogenic LRRK2-mediated trafficking deficits.

Supplementary Materials: The following are available online at http://www.mdpi.com/2073-4409/9/7/1719/s1, containing: Figure S1: Subcellular localization and expression levels of RAB10 variants, Figure S2: Specificity of RAB10 knockdown, Figure S3: Knockdown of RAB10 causes accumulation of EGF in a RAB4-positive compartment rescued upon active RAB7A expression, Figure S4: RAB29 rescues the accumulation of EGF in a RAB4-positive compartment due to G2019S LRRK2 expression, knockdown of RAB8A or knockdown of RAB10, Figure S5: RAB29 recruits G2019S LRRK2 to the Golgi complex upon higher expression levels, Figure S6: Rescue of G2019S LRRK2-mediated EGF trafficking deficits by RAB29 is impaired upon higher expression levels, Figure S7: siRNA-mediated knockdown of RAB29 or transient disruption of Golgi complex integrity by BFA does not affect EGF trafficking, Figure S8: The EGF trafficking deficits mediated by dominant-negative RAB7A are reversed upon RAB29 expression.

Author Contributions: Conceptualization, S.H.; methodology, S.H. and P.R.-R.; validation, B.F. and E.F.; formal analysis, P.R.-R., M.R.-L., B.F. and E.F.; investigation, P.R.-R., M.R.-L., B.F. and E.F.; resources, S.H.; writing - original draft preparation, S.H. and P.R.-R.; writing - review and editing, P.R.-R., M.R.-L., B.F. and E.F.; visualization, S.H., P.R.-R. and M.R.-L.; supervision, S.H.; project administration, S.H.; funding acquisition, S.H. All authors have read and agreed to the published version of the manuscript.

Funding: This work was funded by the Spanish Ministry of Economy and Competitiveness (MINECO) (SAF2017-89402-R) and the Michael J. Fox Foundation (MJFF) (to S.H.). M.R.-L. is enrolled in the PhD program of Biochemistry and Molecular Biology at the University of Granada, and supported by a Fellowship (FPI; BES-2015-075580) from the MINECO.

Acknowledgments: We thank D. Alessi for providing sheep anti-RAB29 antibodies, and L. Montosa for technical assistance with microscopy.

Conflicts of Interest: The authors declare that there are no competing interests.

\section{References}

1. Alessi, D.R.; Sammler, E. LRRK2 kinase in Parkinson's disease. Science 2018, 360, 36-37. [CrossRef] [PubMed]

2. Simón-Sánchez, J.; Schulte, C.; Bras, J.J.M.; Sharma, M.; Gibbs, J.R.; Berg, D.; Paisan-Ruiz, C.; Lichtner, P.; Scholz, S.W.; Hernandez, D.G.; et al. Genome-wide association study reveals genetic risk underlying Parkinson's disease. Nat. Genet. 2009, 41, 1308-1312. [CrossRef] [PubMed]

3. Kluss, J.H.; Mamais, A.; Cookson, M.R. LRRK2 links genetic and sporadic Parkinson's disease. Biochem. Soc. Trans. 2019, 47, 651-661. [CrossRef] [PubMed]

4. Greggio, E.; Jain, S.; Kingsbury, A.; Bandopadhyay, R.; Lewis, P.; Kaganovich, A.; van der Brug, M.P.; Beilina, A.; Blackinton, J.; Thomas, K.J.; et al. Kinase activity is required for the toxic effects of mutant LRRK2/dardarin. Neurobiol. Dis. 2006, 23, 329-341. [CrossRef] [PubMed]

5. Smith, W.W.; Pei, Z.; Jiang, H.; Dawson, V.L.; Dawson, T.M.; Ross, C.A. Kinase activity of mutant LRRK2 mediates neuronal toxicity. Nat. Neurosci. 2006, 9, 1231-1233. [CrossRef]

6. Jaleel, M.; Nichols, R.J.; Deak, M.; Campbell, D.G.; Gillardon, F.; Knebel, A.; Alessi, D.R. LRRK2 phosphorylates moesin at threonine-558: characterization of how Parkinson's disease mutants affect kinase activity. Biochem. J. 2007, 405, 307-317. [CrossRef]

7. West, A.B.; Moore, D.J.; Choi, C.; Andrabi, S.A.; Li, X.; Dikeman, D.; Biskup, S.; Zhang, Z.; Lim, K.L.; Dawson, V.L.; et al. Parkinson's disease-associated mutations in LRRK2 link enhanced GTP-binding and kinase activities to neuronal toxicity. Hum. Mol. Genet. 2007, 16, 223-232. [CrossRef]

8. Luzón-Toro, B.; de la Torre, E.R.; Delgado, A.; Pérez-Tur, J.; Hilfiker, S. Mechanistic insight into the dominant mode of the Parkinson's disease-associated G2019S LRRK2 mutation. Hum. Mol. Genet. 2007, 16, 2031-2039. [CrossRef] 
9. Lee, B.D.; Shin, J.-H.; VanKampen, J.; Petrucelli, L.; West, A.B.; Ko, H.S.; Lee, Y.-I.; Maguire-Zeiss, K.A.; Bowers, W.J.; Federoff, H.J.; et al. Inhibitors of leucine-rich repeat kinase-2 protect against models of Parkinson's disease. Nat. Med. 2010, 16, 998-1000. [CrossRef]

10. Stafa, K.; Trancikova, A.; Webber, P.J.; Glauser, L.; West, A.B.; Moore, D.J. GTPase activity and neuronal toxicity of parkinson's disease-associated LRRK2 is regulated by ArfGAP1. PLoS Genet. 2012, 8. [CrossRef]

11. Hatcher, J.; Choi, H.; Alessi, D.; Gray, N. Small-Molecule Inhibitors of LRRK2. Adv Neurobiol. 2017, 14, 241-264. [CrossRef] [PubMed]

12. Steger, M.; Diez, F.; Dhekne, H.S.; Lis, P.; Nirujogi, R.S.; Karayel, O.; Tonelli, F.; Martinez, T.N.; Lorentzen, E.; Pfeffer, S.R.; et al. Systematic proteomic analysis of LRRK2-mediated rab GTPase phosphorylation establishes a connection to ciliogenesis. Elife 2017, 6, 1-22. [CrossRef] [PubMed]

13. Stenmark, H. Rab GTPases as coordinators of vesicle traffic. Nat. Rev. Mol. Cell Biol. 2009, 10, 513-525. [CrossRef]

14. Wandinger-Ness, A.; Zerial, M. Rab Proteins and the Compartmentalization of the Endosomal System. Cold Spring Harb. Perspect. Biol. 2014, 6, a022616. [CrossRef]

15. Pfeffer, S.R. Rab GTPases: master regulators that establish the secretory and endocytic pathways. Mol. Biol. Cell 2017, 28, 712-715. [CrossRef] [PubMed]

16. Lamber, E.P.; Siedenburg, A.-C.; Barr, F.A. Rab regulation by GEFs and GAPs during membrane traffic. Curr. Opin. Cell Biol. 2019, 59, 34-39. [CrossRef]

17. Itzen, A.; Goody, R.S. GTPases involved in vesicular trafficking: Structures and mechanisms. Semin. Cell Dev. Biol. 2011, 22, 48-56. [CrossRef]

18. Steger, M.; Tonelli, F.; Ito, G.; Davies, P.; Trost, M.; Vetter, M.; Wachter, S.; Lorentzen, E.; Duddy, G.; Wilson, S.; et al. Phosphoproteomics reveals that Parkinson's disease kinase LRRK2 regulates a subset of Rab GTPases. Elife 2016, 5, 1-28. [CrossRef]

19. Madero-Pérez, J.; Fdez, E.; Fernández, B.; Lara Ordóñez, A.J.; Blanca Ramírez, M.; Gómez-Suaga, P.; Waschbüsch, D.; Lobbestael, E.; Baekelandt, V.; Nairn, A.C.; et al. Parkinson disease-associated mutations in LRRK2 cause centrosomal defects via Rab8a phosphorylation. Mol. Neurodegener. 2018, 13, 1-22. [CrossRef]

20. Rivero-Ríos, P.; Romo-Lozano, M.; Fasiczka, R.; Naaldijk, Y.; Hilfiker, S. LRRK2-Related Parkinson's Disease Due to Altered Endolysosomal Biology With Variable Lewy Body Pathology: A Hypothesis. Front. Neurosci. 2020, 14, 556. [CrossRef]

21. Dodson, M.W.; Zhang, T.; Jiang, C.; Chen, S.; Guo, M. Roles of the Drosophila LRRK2 homolog in Rab7-dependent lysosomal positioning. Hum. Mol. Genet. 2012, 21, 1350-1363. [CrossRef] [PubMed]

22. MacLeod, D.A.; Rhinn, H.; Kuwahara, T.; Zolin, A.; Di Paolo, G.; MacCabe, B.D.; Marder, K.S.; Honig, L.S.; Clark, L.N.; Small, S.A.; et al. RAB7L1 Interacts with LRRK2 to Modify Intraneuronal Protein Sorting and Parkinson's Disease Risk. Neuron 2013, 77, 425-439. [CrossRef] [PubMed]

23. Beilina, A.; Rudenko, I.N.; Kaganovich, A.; Civiero, L.; Chau, H.; Kalia, S.K.; Kalia, L.V.; Lobbestael, E.; Chia, R.; Ndukwe, K.; et al. Unbiased screen for interactors of leucine-rich repeat kinase 2 supports a common pathway for sporadic and familial Parkinson disease. Proc. Natl. Acad. Sci. USA 2014, 111, 2626-2631. [CrossRef] [PubMed]

24. Yun, H.J.; Kim, H.; Ga, I.; Oh, H.; Ho, D.H.; Kim, J.; Seo, H.; Son, I.; Seol, W. An early endosome regulator, Rab5b, is an LRRK2 kinase substrate. J. Biochem. 2015, 157, 485-495. [CrossRef]

25. Ito, G.; Katsemonova, K.; Tonelli, F.; Lis, P.; Baptista, M.A.S.; Shpiro, N.; Duddy, G.; Wilson, S.; Ho, P.W.-L.; Ho, S.-L.; et al. Phos-tag analysis of Rab10 phosphorylation by LRRK2: a powerful assay for assessing kinase function and inhibitors. Biochem. J. 2016, 473, 2671-2685. [CrossRef]

26. Thirstrup, K.; Dächsel, J.C.; Oppermann, F.S.; Williamson, D.S.; Smith, G.P.; Fog, K.; Christensen, K.V. Selective LRRK2 kinase inhibition reduces phosphorylation of endogenous Rab10 and Rab12 in human peripheral mononuclear blood cells. Sci. Rep. 2017, 7, 1-18. [CrossRef]

27. Beilina, A.; Bonet-Ponce, L.; Kumaran, R.; Kordich, J.J.; Ishida, M.; Mamais, A.; Kaganovich, A.; Saez-Atienzar, S.; Gershlick, D.C.; Roosen, D.A.; et al. The Parkinson's Disease Protein LRRK2 Interacts with the GARP Complex to Promote Retrograde Transport to the trans-Golgi Network. Cell Rep. 2020, 31, 107614. [CrossRef]

28. Katzmann, D.J.; Odorizzi, G.; Emr, S.D. Receptor downregulation and multivesicular-body sorting. Nat. Rev. Mol. Cell Biol. 2002, 3, 893-905. [CrossRef] 
29. Chi, S.; Cao, H.; Wang, Y.; McNiven, M.A. Recycling of the epidermal growth factor receptor is mediated by a novel form of the clathrin adaptor protein Eps15. J. Biol. Chem. 2011, 286, 35196-35208. [CrossRef]

30. Gómez-Suaga, P.; Rivero-Ríos, P.; Fdez, E.; Blanca Ramírez, M.; Ferrer, I.; Aiastui, A.; López De Munain, A.; Hilfiker, S. LRRK2 delays degradative receptor trafficking by impeding late endosomal budding through decreasing Rab7 activity. Hum. Mol. Genet. 2014, 23, 6779-6796. [CrossRef]

31. Rivero-Ríos, P.; Romo-Lozano, M.; Madero-Pérez, J.; Thomas, A.P.; Biosa, A.; Greggio, E.; Hilfiker, S. The G2019S variant of leucine-rich repeat kinase 2 (LRRK2) alters endolysosomal trafficking by impairing the function of the GTPase RAB8A. J. Biol. Chem. 2019, 294, 4738-4758. [CrossRef]

32. Fernández, B.; Lara Ordóñez, A.J.; Fdez, E.; Mutez, E.; Comptdaer, T.; Leghay, C.; Kreisler, A.; Simonin, C.; Vandewynckel, L.; Defebvre, L.; et al. Centrosomal cohesion deficits as cellular biomarker in lymphoblastoid cell lines from LRRK2 Parkinson's disease patients. Biochem. J. 2019, 476, 2797-2813. [CrossRef]

33. Lis, P.; Burel, S.; Steger, M.; Mann, M.; Brown, F.; Diez, F.; Tonelli, F.; Holton, J.L.; Ho, P.W.; Ho, S.-L.; et al. Development of phospho-specific Rab protein antibodies to monitor in vivo activity of the LRRK2 Parkinson's disease kinase. Biochem. J. 2018, 475, 1-22. [CrossRef] [PubMed]

34. Fan, Y.; Howden, A.J.M.; Sarhan, A.R.; Lis, P.; Ito, G.; Martinez, T.N.; Brockmann, K.; Gasser, T.; Alessi, D.R.; Sammler, E.M. Interrogating Parkinson's disease LRRK2 kinase pathway activity by assessing Rab10 phosphorylation in human neutrophils. Biochem. J. 2018, 475, 23-44. [CrossRef]

35. Atashrazm, F.; Hammond, D.; Perera, G.; Bolliger, M.F.; Matar, E.; Halliday, G.M.; Schüle, B.; Lewis, S.J.G.; Nichols, R.J.; Dzamko, N. LRRK2-mediated Rab10 phosphorylation in immune cells from Parkinson's disease patients. Mov. Disord. 2019, 34, 406-415. [CrossRef] [PubMed]

36. Zhang, Y.; Sloan, S.A.; Clarke, L.E.; Caneda, C.; Plaza, C.A.; Blumenthal, P.D.; Vogel, H.; Steinberg, G.K.; Edwards, M.S.B.; Li, G.; et al. Purification and Characterization of Progenitor and Mature Human Astrocytes Reveals Transcriptional and Functional Differences with Mouse. Neuron 2016, 89, 37-53. [CrossRef] [PubMed]

37. Babbey, C.M.; Ahktar, N.; Wang, E.; Chih-Hsiung Cheng, C.; Grant, B.D.; Dunn, E.W. Rab10 Regulates Membrane Transport through Early Endosomes of Polarized Madin-Darby Canine Kidney Cells. Mol. Biol. Cell 2006, 17, 3156-3175. [CrossRef] [PubMed]

38. Roland, J.T.; Lapierre, L.A.; Goldenring, J.R. Alternative Splicing in Class V Myosins Determines Association with Rab10. J. Biol. Chem. 2009, 284, 1213-1223. [CrossRef] [PubMed]

39. Lara Ordónez, A.J.; Fernández, B.; Fdez, E.; Romo-Lozano, M.; Madero-Pérez, J.; Lobbestael, E.; Baekelandt, V.; Aiastui, A.; López de Munaín, A.; Melrose, H.L.; et al. RAB8, RAB10 and RILPL1 contribute to both LRRK2 kinase-mediated centrosomal cohesion and ciliogenesis deficits. Hum. Mol. Genet. 2019, 28, 3552-3568. [CrossRef]

40. Etoh, K.; Fukuda, M. Rab10 regulates tubular endosome formation through KIF13A and KIF13B motors. J. Cell Sci. 2019, 132, jcs226977. [CrossRef]

41. Chua, C.E.L.; Tang, B.L. Rab 10-A traffic controller in multiple cellular pathways and locations. J. Cell. Physiol. 2018, 233, 6483-6494. [CrossRef] [PubMed]

42. Sato, T.; Iwano, T.; Kunii, M.; Matsuda, S.; Mizuguchi, R.; Jung, Y.; Hagiwara, H.; Yoshihara, Y.; Yuzaki, M.; Harada, R.; et al. Rab8a and Rab8b are essential for several apical transport pathways but insufficient for ciliogenesis. J. Cell Sci. 2014, 127, 422-431. [CrossRef] [PubMed]

43. Schuck, S.; Gerl, M.J.; Ang, A.; Manninen, A.; Keller, P.; Mellman, I.; Simons, K. Rab10 is Involved in Basolateral Transport in Polarized Madin-Darby Canine Kidney Cells. Traffic 2007, 8, 47-60. [CrossRef] [PubMed]

44. Homma, Y.; Fukuda, M. Rabin8 regulates neurite outgrowth in both GEF activity-dependent and -independent manners. Mol. Biol. Cell 2016, 27, 2107-2118. [CrossRef] [PubMed]

45. Tao, T.; Sun, J.; Peng, Y.; Li, Y.; Wang, P.; Chen, X.; Zhao, W.; Zheng, Y.-Y.; Wei, L.; Wang, W.; et al. Golgi-resident TRIO regulates membrane trafficking during neurite outgrowth. J. Biol. Chem. 2019, 294, 10954-10968. [CrossRef] [PubMed]

46. Tucci, A.; Nalls, M.A.; Houlden, H.; Revesz, T.; Singleton, A.B.; Wood, N.W.; Hardy, J.; Paisán-Ruiz, C. Genetic variability at the PARK16 locus. Eur. J. Hum. Genet. 2010, 18, 1356-1359. [CrossRef] [PubMed]

47. Gan-Or, Z.; Bar-Shira, A.; Dahary, D.; Mirelman, A.; Kedmi, M.; Gurevich, T.; Giladi, N.; Orr-Urtreger, A. Association of Sequence Alterations in the Putative Promoter of RAB7L1 With a Reduced Parkinson Disease Risk. Arch. Neurol. 2012, 69, 105-110. [CrossRef] 
48. Guo, X.-Y.; Chen, Y.-P.; Song, W.; Zhao, B.; Cao, B.; Wei, Q.-Q.; Ou, R.-W.; Yang, Y.; Yuan, L.-X.; Shang, H.-F. An association analysis of the rs1572931 polymorphism of the RAB7L1 gene in Parkinson's disease, amyotrophic lateral sclerosis and multiple system atrophy in China. Eur. J. Neurol. 2014, 21, 1337-1343. [CrossRef]

49. Pihlstrøm, L.; Rengmark, A.; Bjørnarå, K.A.; Dizdar, N.; Fardell, C.; Forsgren, L.; Holmberg, B.; Larsen, J.P.; Linder, J.; Nissbrandt, H.; et al. Fine mapping and resequencing of the PARK16 locus in Parkinson's disease. J. Hum. Genet. 2015, 60, 357-362. [CrossRef]

50. Khaligh, A.; Goudarzian, M.; Moslem, A.; Mehrtash, A.; Jamshidi, J.; Darvish, H.; Emamalizadeh, B. RAB7L1 promoter polymorphism and risk of Parkinson's disease; a case-control study. Neurol. Res. 2017, 39, 468-471. [CrossRef]

51. He, T.; Wang, J.; Wang, X.; Deng, W.; Jiang, H.; Xie, J.; Sun, P. Association between PARK16 and Parkinson's disease: A meta-analysis. Neurosci. Lett. 2017, 657, 179-188. [CrossRef] [PubMed]

52. Goudarzian, M.; Khaligh, A.; Fourozan, R.; Jamal Mirmoosavi, S.; Darvish, H.; Safaralizadeh, T.; Emamalizadeh, B. The rs1572931 polymorphism of the RAB7L1 gene promoter is associated with reduced risk of Parkinson's disease. Neurol. Res. 2015, 37, 1029-1031. [CrossRef] [PubMed]

53. Soto-Ortolaza, A.I.; Heckman, M.G.; Labbé, C.; Serie, D.J.; Puschmann, A.; Rayaprolu, S.; Strongosky, A.; Boczarska-Jedynak, M.; Opala, G.; Krygowska-Wajs, A.; et al. GWAS risk factors in Parkinson's disease: LRRK2 coding variation and genetic interaction with PARK16. Am. J. Neurodegener. Dis. 2013, 2, $287-299$. [PubMed]

54. Liu, Z.; Bryant, N.; Kumaran, R.; Beilina, A.; Abeliovich, A.; Cookson, M.R.; West, A.B. LRRK2 phosphorylates membrane-bound Rabs and is activated by GTP-bound Rab7L1 to promote recruitment to the trans-Golgi network. Hum. Mol. Genet. 2018, 27, 385-395. [CrossRef] [PubMed]

55. Madero-Pérez, J.; Fernández, B.; Lara Ordóñez, A.J.; Fdez, E.; Lobbestael, E.; Baekelandt, V.; Hilfiker, S. RAB7L1-Mediated Relocalization of LRRK2 to the Golgi Complex Causes Centrosomal Deficits via RAB8A. Front. Mol. Neurosci. 2018, 11, 417. [CrossRef]

56. Purlyte, E.; Dhekne, H.S.; Sarhan, A.R.; Gomez, R.; Lis, P.; Wightman, M.; Martinez, T.N.; Tonelli, F.; Pfeffer, S.R.; Alessi, D.R. Rab29 activation of the Parkinson's disease-associated LRRK2 kinase. EMBO J. 2018, 37, 1-18. [CrossRef]

57. Gomez, R.C.; Wawro, P.; Lis, P.; Alessi, D.R.; Pfeffer, S.R. Membrane association but not identity is required for LRRK2 activation and phosphorylation of Rab GTPases. J. Cell Biol. 2019, 218, 4157-4170. [CrossRef]

58. Civiero, L.; Vancraenenbroeck, R.; Belluzzi, E.; Beilina, A.; Lobbestael, E.; Reyniers, L.; Gao, F.; Micetic, I.; de Maeyer, M.; Bubacco, L.; et al. Biochemical Characterization of Highly Purified Leucine-Rich Repeat Kinases 1 and 2 Demonstrates Formation of Homodimers. PLoS ONE 2012, 7. [CrossRef]

59. Peralta, E.R.; Martin, B.C.; Edinger, A.L. Differential effects of TBC1D15 and mammalian Vps39 on Rab7 activation state, lysosomal morphology, and growth factor dependence. J. Biol. Chem. 2010, 285, 16814-16821. [CrossRef]

60. Ceresa, B.P.; Bahr, S.J. Rab7 activity affects epidermal growth factor:epidermal growth factor receptor degradation by regulating endocytic trafficking from the late endosome. J. Biol. Chem. 2006, 281, 1099-1106. [CrossRef]

61. Vanlandingham, P.A.; Ceresa, B.P. Rab7 Regulates Late Endocytic Trafficking Downstream of Multivesicular Body Biogenesis and Cargo Sequestration. J. Biol. Chem. 2009, 284, 12110-12124. [CrossRef] [PubMed]

62. Rassu, M.; Grazia, M.; Giudice, D.; Sanna, S.; Taymans, J.M.; Morari, M.; Brugnoli, A.; Frassineti, M.; Masala, A.; Esposito, S.; et al. Role of LRRK2 in the regulation of dopamine receptor trafficking. PLoS ONE 2017, 12. [CrossRef]

63. Heaton, G.R.; Landeck, N.; Mamais, A.; Nalls, M.A.; Nixon-Abell, J.; Kumaran, R.; Beilina, A.; Pellegrini, L.; Li, Y.; Harvey, K.; et al. Sequential screening nominates the Parkinson's disease associated kinase LRRK2 as a regulator of Clathrin-mediated endocytosis. Neurobiol. Dis. 2020, 141, 104948. [CrossRef]

64. McCaffrey, M.W.; Bielli, A.; Cantalupo, G.; Mora, S.; Roberti, V.; Santillo, M.; Drummond, F.; Bucci, C. Rab4 affects both recycling and degradative endosomal trafficking. FEBS Lett. 2001, 495, 21-30. [CrossRef]

65. Braun, A.C.; Hendrick, J.; Eisler, S.A.; Schmid, S.; Hausser, A.; Olayioye, M.A. The Rho-specific GAP protein DLC3 coordinates endocytic membrane trafficking. J. Cell Sci. 2015, 128, 1386-1399. [CrossRef] 
66. Goueli, B.S.; Powell, M.B.; Finger, E.C.; Pfeffer, S.R. TBC1D16 is a Rab4A GTPase activating protein that regulates receptor recycling and EGF receptor signaling. Proc. Natl. Acad. Sci. USA 2012, 109, 15787-15792. [CrossRef]

67. Bucci, C.; Thomsen, P.; Nicoziani, P.; McCarthy, J.; van Deurs, B. Rab7: a key to lysosome biogenesis. Mol. Biol. Cell 2000, 11, 467-480. [CrossRef]

68. Yu, L.; McPhee, C.K.; Zheng, L.; Mardones, G.A.; Rong, Y.; Peng, J.; Mi, N.; Zhao, Y.; Liu, Z.; Wan, F.; et al. Termination of autophagy and reformation of lysosomes regulated by mTOR. Nature 2010, 465, 942-946. [CrossRef] [PubMed]

69. Hutagalung, A.H.; Novick, P.J. Role of Rab GTPases in Membrane Traffic and Cell Physiology. Physiol. Rev. 2011, 91, 119-149. [CrossRef]

70. Pfeffer, S.R. Rab GTPase regulation of membrane identity. Curr. Opin. Cell Biol. 2013, 25, 414-419. [CrossRef] [PubMed]

71. Barr, F.A. Review series: Rab GTPases and membrane identity: causal or inconsequential? J. Cell Biol. 2013, 202, 191-199. [CrossRef] [PubMed]

72. Langemeyer, L.; Perz, A.; Kümmel, D.; Ungermann, C. A guanine nucleotide exchange factor (GEF) limits Rab GTPase-driven membrane fusion. J. Biol. Chem. 2018, 293, 731-739. [CrossRef] [PubMed]

73. Langemeyer, L.; Borchers, A.-C.; Herrmann, E.; Füllbrunn, N.; Han, Y.; Perz, A.; Auffarth, K.; Kümmel, D.; Ungermann, C. A conserved and regulated mechanism drives endosomal Rab transition. Elife 2020, 9, e56090. [CrossRef] [PubMed]

74. Kuwahara, T.; Inoue, K.; D’Agati, V.D.; Fujimoto, T.; Eguchi, T.; Saha, S.; Wolozin, B.; Iwatsubo, T.; Abeliovich, A. LRRK2 and RAB7L1 coordinately regulate axonal morphology and lysosome integrity in diverse cellular contexts. Sci. Rep. 2016, 6, 29945. [CrossRef]

75. Onnis, A.; Finetti, F.; Patrussi, L.; Gottardo, M.; Cassioli, C.; Spanò, S.; Baldari, C.T. The small GTPase Rab29 is a common regulator of immune synapse assembly and ciliogenesis. Cell Death Differ. 2015, 22, 1687-1699. [CrossRef]

(C) 2020 by the authors. Licensee MDPI, Basel, Switzerland. This article is an open access article distributed under the terms and conditions of the Creative Commons Attribution (CC BY) license (http://creativecommons.org/licenses/by/4.0/). 